UCRL -8040

Physics and

Mathematics

\title{
UNIVERSITY OF CALIFORNIA.
}

Radiation Laboratory

Berkeley, California

Contract No. W-7405-eng-48

\section{DISINTEGRATION OF HELIUM BY 300-Mev NEUTRONS \\ William H. Innes}

(Thesis)

November 1957

Printed for the U. S. Atomic Energy Commission 


\section{DISCLAIMER}

This report was prepared as an account of work sponsored by an agency of the United States Government. Neither the United States Government nor any agency Thereof, nor any of their employees, makes any warranty, express or implied, or assumes any legal liability or responsibility for the accuracy, completeness, or usefulness of any information, apparatus, product, or process disclosed, or represents that its use would not infringe privately owned rights. Reference herein to any specific commercial product, process, or service by trade name, trademark, manufacturer, or otherwise does not necessarily constitute or imply its endorsement, recommendation, or favoring by the United States Government or any agency thereof. The views and opinions of authors expressed herein do not necessarily state or reflect those of the United States Government or any agency thereof. 


\section{DISCLAIMER}

Portions of this document may be illegible in electronic image products. Images are produced from the best available original document. 


\section{Contents}

Abstract

I. Introduction

II. Experimental Procedure

1. Apparatus . . . . . . . . . . . . . . 8

2. Operation . . . . . . . . . . . . . . . . .

3. Photography . . . . . . . . . . . . . . . 9

III. Method of Analysis of Events , . . . . . . . . . . 10

1. Available Dáta . . . . . . . . . . . . . 10

2. Identification Procedure . . . . . . . . . . . 11

3. Errors in Measurement . . . . . . . . . . 15

4. Calculations . . . . . . . . . . . . . . 17

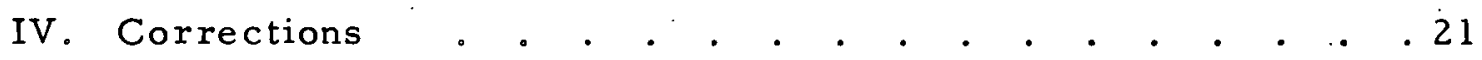

1. Single-Pronged Stars . . . . . . . . . . . 21

2. Two-Pronged Stars . . . . . . . . . . . 22

V. Results and Discussion

1. Inelastic Events . . . . . . . . . . . . . 24

2. Elastic Events . . . . . . . . . . . . . 26

3. Meson Events . . . . . . . . . . . . . 29

4. Cross Sections . . . . . . . . . . . . . 29

5. Errors... . . . . . . . . . . . . 31

6. Comparison with Theory . . . . . . . . . . . . . 31

7. Comparison with Other Experiments . . . . . . . 34

8. Energy and Angular Distributions .. . . . . . . . 40 


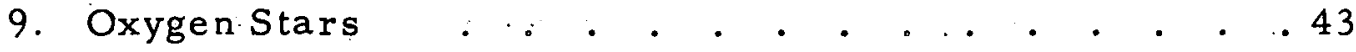

10. Azimuthal Symmetry Check. . . . . . . . . 45

VI. Conclusions . . . . . . . . . . . . . . . . . . 46

VII... Acknowledgments . . . . . . . . . . . . . . . . . . 48

Appendix

I. Definitions . . . . . . . . . . . . . . . 49

II. Derivation of Formulas . . . . . . . . . . . . . 50

Figures . . . . . . . . . . . . . . . . . . . . . . . 54

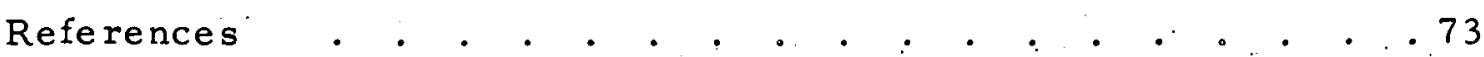




\title{
William $\mathrm{H}$. Innes \\ Radiation Laboratory \\ University of California \\ Berkeley, California
}

DISINTEGRATION OF HELIUM BY 300-Mev NEUTRONS

November 1957

\begin{abstract} reactions are:

$$
\begin{array}{ll}
\text { Inelastic } & \mathrm{He}^{4}(n, \mathrm{pn}) \mathrm{t}, \\
& \mathrm{He}^{4}(n, d) t, \\
& \mathrm{He}^{4}(n, 2 n p) d, \\
& \mathrm{He}^{4}(n, d n) d, \\
& \mathrm{He}^{4}(n, 2 n 2 p) n, \\
& \mathrm{He}^{4}(n, 2 n) \mathrm{He}^{3} ; \\
\text { Elastic } & \mathrm{He}^{4}(n, n) \mathrm{He}^{4} .
\end{array}
$$
\end{abstract}

An investigation of the inelastic and elastic reactions between high-energy neutrons and helium nuclei has been conducted with a cloud chamber filled with helium and operating in a pulsed magnetic field of 21,700 gauss. Neutrons produced by bombardment of a $1 / 2$-inch LiD target with 340-Mev protons in the 184-inch synchrocyclotron were collimated and passed through the 22 -inch expansion cloud chamber, which was filled with helium gas to a total pressure of $89.8 \mathrm{~cm} \mathrm{Hg}$. Exclusive of meson-producing reactions, the possible

The total number of events, for incident neutrons above $160 \mathrm{Mev}$, was normalized to the interpolated $\mathrm{n}-\mathrm{He}^{4}$ total cross section at $300 \mathrm{Mev}$, and absolute cross sections for the various processes were established. Energy and angular distributions of the reaction products have been 


$$
-5-
$$

compared with available theoretical predictions and other experiments. A few cases of meson production were noted. 


\section{INTRODUCTION}

There exists today a wealth of experimental data pertaining to. the interactions of high-energy nucleons with other nucleons, simple nuclei, and complex nuclei. This information, much of which is concerned with neutron and proton interactions with nuclei ranging from the very lightest to the very heaviest, embraces incident nucleon energies ranging from a few Mev to the several-Bev energies of the Bevatron and the Cosmotron.

Despite thị great amount and variety of data, or perhaps as a consequence, the outstanding problem of nuclear physics at the present time is the formulation of a satisfactory fundamental theory of nuclear forces--satisfactory in the sense that it not only unambiguously explains all the observed results but also can predict additional phenom: ena whose pursuit will lead to the orderly and rapid advance of nuclear science.

Increasing emphasis is being placed on experiments that may lead to an understanding of these forces. One of the most fruitful approaches in establishing models of the nucleus, and in revealing the behavior of several nucleons in close proximity, has been in scattering experiments with light nuclei, in which only a few nucleons are involved. As has been pointed out by Tannenwald, ${ }^{1}$ the disintegration of helium presents an unique case because, while on the one hand there are so few particles involved that a theoretical analysis of the interactions between individual nucleons may be hoped for, it can also on the other hand show some of the properties of heavier nuclei, owing to the tightly bound structure of the helium nucleus. In particular, if the alpha particle indeed exists as a substructure in heavier nuclei, then helium disintegration will be of value in interpreting the structure and disintegration of heavier nuclei.

The identity, frequency of occurrence, and distributions in angle and energy of the secondary particles emitted in the disintegration of helium by high-energy neutrons are therefore expected to be useful in analyses of nuclear structure and of the nature of nuclear forces. For energies above a few Mev, however, there appears to be little 
information in this area. Tracy and Powell, using a cloud chamber containing a mixture of helium and oxygen in a magnetic field of 13, 000 gauss, studied secondary particles emitted under bombardment by $90-\mathrm{Mev}$ neutrons; ${ }^{2}$ analysis of helium data was complicated by the presence of large numbers of oxygen nuclei. Swartz, using a cloud chamber containing only helium and a small amount of water vapor, but without a magnetic field, studied secondary particles under bombardment by $200-\mathrm{Mev}$ neutrons; ${ }^{3}$ analysis of the data was complicated by the absence of a magnetic field. Tannenwald, using a cloud chamber containing only helium and a small amount of water vapor in a magnetic field of 22,000 gauss, studied helium disintegration by $90-\mathrm{Mev}$ neutrons; the absence of any significant number of other nuclei, and the use of a strong magnetic field, enabled him to make the first complete and detailed analysis of the identity and characteristics of secondary particles emitted in high-energy neutron bombardment of helium. Hillman, Stahl, and Ramsey have measured the total cross section of liquefied helium for 48 -Mev and 88 -Mev neutrons. 4 Moulthrop, using a highpressure diffusion cloud chamber in a magnetic field of 21,000 gauss, studied negative pion production in the bombardment of helium by 300-Mev neutrons. ${ }^{5}$ Theoretical predictions concerning the disintegration of helium by high-energy neutrons are also limited. Heidmann has analyzed the neutron-helium scattering problem, for incident neutron energies of $90 \mathrm{Mev}$ and $200 \mathrm{Mev}$, using the Born approximation and Gaussian potentials and wave functions. 6 ,

The experiment presented herein extends the work of Tannenwald ard Swartz to higher energies and, in a sense, supplements that of Moulthrop, who limited his complete analysis to those interactions which resulted in the production of pions. The cloud chamber is particularly adapted to studies of this type, as it presents the ultimate. in "thin" targets, resulting in the best possible view of the associated particles in the reaction. 


\section{II . EXPERIMENTAL PROCEDURE}

\section{Apparatus}

The neutrons produced by bombarding a $1 / 2$-inch-thick LiD target with 340-Mev protons were collimated inside, through, and outside the concrete shielding of the 184-inch Berkeley synchrocyclotron by a 4-stage collimation system (see Figs. 13 and 14). The tapered lead collimator, inside the concrete shielding, was circular in cross section and 86.5 inches long, and had an exit diameter of 6 inches; the hole in the concrete shielding was circular in cross section, 208.0 inches long, with exit diameter of 12 inches; the first copper collimator. in the concrete shielding, was rectangular in cross section, $23 / 32$ by $2-3 / 8$ inches, and 34 inches long; the second (final) copper collimator, outside the concrete shielding, was rectangular in cross section, 1 by 3 inches, and 34 inches long. The neutrons entered the cloud chamber through a 1 - by-5-inch copper foil window, 3 mils thick; and passed out through a similar window to reduce backscattering from the exit wall of the chamber.

The Wilson cloud chamber used was developed by Powell 8 and has been used by him and others for a number of experiments. It is 22 inches in diameter, has a sensitive region $3-1 / 2$ inches deep, and has a pressure-controlled expansion ratio. It fits into the 6 -inch gap of a magnet capable of producing a pulsed field of 21,700 gauss. ${ }^{8}$ The bottom of the chamber is a rubber-covered $1 / 2$-inch-thick lucite disk which moves vertically and is controlled by a pantograph which keeps it accurately horizontal during the expansions. Gelatin containing a black dye covered the disk to a depth of $1 / 16$ inch, providing a black background for track photography. General Electric FT 422 flash tubes were used on opposite sides of the chamber, providing uniform illumination over $2-1 / 2$ inches of the $3-1 / 2$-inch sensitive region. 9 The lamps were simultaneously flashed by the discharge, through each, of 512 -microfarad condensers charged to a potential of 1700 volts. 


\section{Operation}

The cloud chamber was operated in a pulsed magnetic field of 21,700 gauss which was energized by a current pulse of 4000 amperes supplied by a 150-hp generator with a 5-ton flywheel. The field requires about 2.5 seconds to attain its maximum value, where it remains steady for about 0.15 second before being turned off. The chamber was operated on a 1-minute automatically controlled cycle, as follows: the magnet current is turned on in advance so that its maximum coincides with the full expansion of the chamber; the cyclotron beam is pulsed through the chamber at the instant the moving diaphragm hits bottom, and the lights are flashed about 0.04 second after this. The current that passes through the magnet is recorded with each picture by an auxiliary lens which views a magnet-current meter. A clearing field of about 100 volts is removed just prior to expansion of the chamber and turned on again after the lights have flashed.

The chamber was filled with helium gas to a total pressure of $89.8 \mathrm{~cm} \mathrm{Hg}$ in the expanded position; of this pressure, $1.7 \mathrm{~cm}$ was due to the partial vapor pressure of the water in the gelatin. The chamber was then compressed to a total pressure of about $103.0 \mathrm{~cm}$, representing an expansion ratio of around 15\%. The chamber and flash lights were surrounded by a felt-lined box and the whole kept at a constant temperature of $19.3^{\circ} \mathrm{C}$ by means of a temperature-controlled refrigerated water systein.

\section{Photography}

A conical hole in the top pole piece of the magnet permits an automatic motor-driven camera to look down into the chamber and take paired stereoscopic photographs through twin $50-\mathrm{mm} \mathrm{f} / 2$ Leitz Summitar lenses spaced 4-1/2 inches apart. The camera is mounted 27 inches above the top glass of the chamber in a lighttight dome. Photographs are taken in sequence on 100-foot.rolls of 1.8 -inch-wide Eastman Linagraph Ortho film, which is developed to maximum contrast. Since the camera had no shutter, the length of exposure was determined by the length of the flash, about 100 microseconds. 
All counting and measurement of events was made from the ster eoscopic photographs. These were reprojected in the stereoscopic projector (diagrammed in Fig. 15). This projector has been developed for the general use of the Radiation Laboratory cloud chamber group, under Professor Wilson M. Powell; its construction and operation have been described elsewhere. ${ }^{10}$ The projector duplicates the optical system of the camera-cloud chamber arrangement and, using the camera lenses, permits the reconstruction in space of events that occurred in the cloud chamber. This is accomplished by bringing the two track images into alignment on the translucent screen. The screen has three translational and two rotational degrees of freedom for this purpose.

\section{METHOD OF ANALYSIS OF EVENTS}

The use of a cloud chamber in studying nuclear reactions offers several advantages: a pure target can be used; the large solid angle of observation permits the detection of particles of all angles and energies; individual events can be studied in detail and, with the aid of a stereoscopic camera and projector, each charged-particle track can be reproduced in space in its original size, shape, and position; with the addition of a magnetic field, the momentum and energy of each charged particle can be determined. There are also limitations, chief of which is the relatively slow accumulation of data owing to the low target density.

\section{Available Data}

Following an outline by J. Tracy, ${ }^{11}$ the data available for analyzing an event in this investigation may be divided into three categories. These are:

General Experimental Data. This includes knowledge of the direction and approximate energy distribution of the incident neutron beam, the direction and strength of the magnetic field, and the composition and stopping power of the gas mixture in the cloud chamber. 
Individual Star Data. This includes information obtained from measurements on the individual tracks, such as initial radius of curvatire, density, initial direction, range, rate of change of curvature, and rate of change of density.

Auxiliary Information. This includes application of the laws of conservation of momentum, total energy, and charge, as well as knowlèdge: of range-energy relations, specific ionization vs energy relations, and characteristic track endings.

\section{Identification Procedure}

A comprehensive discussion of the ways in which the data outlined above may bc ueed to identify charged particles in cloud chambers is available elsewhere. $1,11,12$ There follows only a brief discussion of the particles here involved and of special situations whose existence sometimes enhanced the usual identification procedures.

Exclusive of meson-producing events, the possible reactions when a neutron strikes a helium nucleus are as follows. The brief symbol that appears on the right is used $\ldots z: \therefore . .$. to represent the reaction to which it corresponds.

Inelastic Reactions

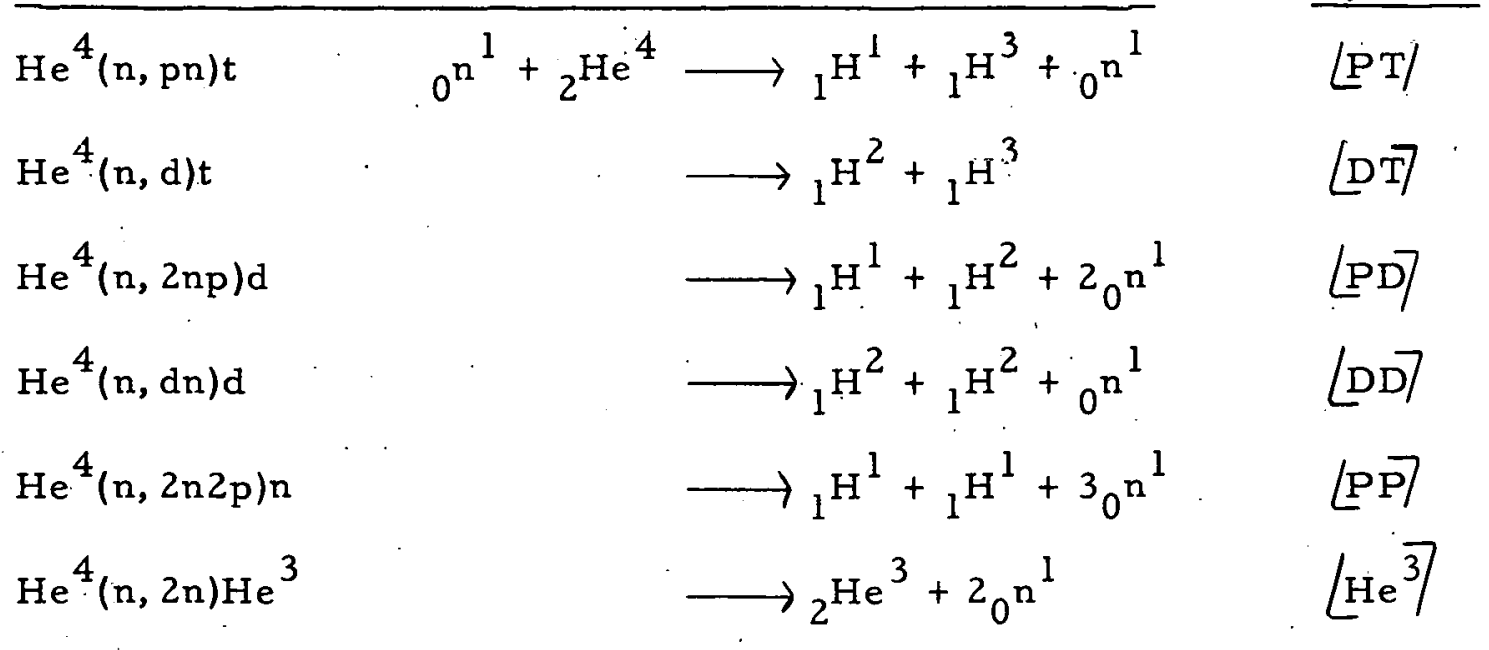

Elastic Reactions
$\mathrm{He}^{4}(n, n) \mathrm{He}^{4}$
$\longrightarrow{ }_{2} \mathrm{He}^{4}+{ }_{0} \mathrm{n}^{1}$
$\left\lfloor\mathrm{He}^{4}\right]$ 
Analysis of an event involving the helium nucleus thus requires. the identification of protons, deuterons, and tritons for two-pronged stars or the identification of $\mathrm{He}^{3 / \mathrm{s}}$ and $\mathrm{He}^{4}$ 's for one-pronged stars. Stars with three or more prongs are observed occasionally; they are due either to oxygen nuclei in the water vapor or to meson-production events with helium or oxygen. The relative number of oxygen nuclei can be computed from the partial pressures existing in the chamber, and in a subsequent section this is compared with the relative numbers of helium and oxygen stars observed. A few cases of meson production were noted; these also are discussed in a later section.

Two-Pronged Stars. The identification of the particles involved in the two-pronged stars rests mainly on measurements of their radii of curvature and an estimate of their relative ionizations. The fact that [DT] is a two-body problem requires that the two prongs be coplanar with the beam direction and that their transverse momenta be equal in magnitude and opposite in direction. These criteria can be used to fairly definitely establish an event as $[\mathrm{DT}]$ when verified by an alternate method of determining the energy of the incident neutron. They can be used, alone, more definitely to rule out $[D T]$ : if one prong is definitely established as a deuteron (or as a triton) and the two prongs are not coplanar with the beam, then the other prong cannot be a triton (or a deuteron); similar reasoning applies if the transverse momenta do not sum to zero within reasonable limits.

It has been possible to identify the particles in the two-pronged stars in most cases. Out of 178 two-pronged stars that satisfied the ultimate selection criteria (dip angles within $\pm 50^{\circ}$ of the horizontal plane; incident-neutron energy equal to or greater than $160 \mathrm{Mev}$ ), 18 were not resolved with certainty during the film-reading process. Of these, 15 were recorded as either $\angle \mathrm{PD}]$ or $\angle \mathrm{PT}]$ and three as either $\angle \mathrm{PD}]$ or $\angle \mathrm{DD}]$. These were resolved, after calculations for the incident-neutron energy for the alternative assumptions and study of the original data, as $11\langle\mathrm{PD}\rangle, 4\langle\mathrm{PT}\rangle$, and $3 \angle \mathrm{DD}\rangle$. If only these three types of events were considered, events (unweighted) were $66 \%\lfloor\mathrm{PT}\rangle, 24 \%\lfloor\mathrm{PD}\rangle$, and $10 \%\lfloor\mathrm{DD}\rangle$ before resolution, and $62 \%$, 
$28 \%$, and $11 \%$ respectively, after the resolution.

One-Pronged Stars. Measurement of radius of curvature coupled with estimates of relative ionization was generally of little value in differ entiating between $\mathrm{He}^{3}$ and $\mathrm{He}^{4}$. The tracks were most frequently of low energy, very dense, and often quite short. Their ionization, relative to minimum, falls in the range above 100 , where estimates of relative density are insufficiently accurate; for the same radius of rurvature the ionization of $\mathrm{He}^{4}$ is only $50 \%$ greater than that of $\mathrm{He}^{3}$. Track's that ended in the chamber, willı sufficicnt range, were identıfied by characteristic endings, and confirmed by comparison of observed and calculated ranges. When the track did not end in the chamber its change of radius with residual rauge was only rarely sufficient to effect a positive determination. Because of the large energy spread of the incident neutrons thereare no unique energy-angle conditions that distinguish the particles with certainty. If the track goes backward in the chamber it cannot be an $\mathrm{He}^{4}$ recoil associated with an incident neutron in the forward direction; this method of resolution also was rarely applicable. A track might be identified as $\mathrm{He}^{3}$ on the basis that such choice results in a reasonable value for the energy of the incident neutron, whereas identification as $H e^{4}$ would result in a neutron energy so large as to be completely unexplainable by errors in measurement.

Less than half the single tracks were identifiable with certainty during the film-reading process. Out of 66 one-pronged stars that satisfied the ultimate selection criteria (dip angles within $\pm 50^{\circ}$ of the horizontal plane; incident-neutron energy equal to or greater than $160 \mathrm{Mev}$ ) only 27 were identified. The results, based on the above outlined considerations, are summarized below:

Characteristic endings

Radius change-residual range

Backward tracks

$E_{n}$ excessive as $\mathrm{He}^{4}$

$\begin{array}{rr}\frac{\mathrm{He}^{4}}{21} & \frac{\mathrm{He}^{3}}{0} \\ 3 & 1 \\ 0 & 1 \\ \frac{0}{24} & \frac{1}{3}\end{array}$


Less than one-third were identified by characteristic endings; in comparison, Tannenwald was able to so identify about one-half at $90 \mathrm{Mev}$. No $\mathrm{He}^{-3}$ that on calculation satisfied the neutron-energy criterion were observed to end in the chamber; a few apparent ones, associated with neutrons of lower energy, had been so identified. At $90 \mathrm{Mev}$ Tannenwald found only two, compared with $139 \mathrm{He}^{4}$.

Apportionment of the 39 unidentified tracks remaining, of the 66 here considered, in the ratios of the above table would yield 35 as $\mathrm{He}^{4}$ and 4 as $\mathrm{He}^{3}$. On the basis of a careful consideration of the resultant incident-neutron energies (minimum only in the case of $\mathrm{He}^{3}$ ) when each of these 39 events was calculated as $\mathrm{He}^{3}$ and as $\mathrm{He}^{4}$, and of the similar results pertinent to the much greater number (454) of single-pronged events that resulted in a neutron energy of less than $160 \mathrm{Mev}$ on either assumption, the final apportionment made was 36 to $\mathrm{He}^{4}$ and 3 to $\mathrm{He}^{3}$.

A table of ionization (relative to minimum) vs magnetic rigidity for protons, deuterons, tritons, $\mathrm{He}^{3}$, and $\mathrm{He}^{4}$, prepared by Donald. Johnson, ${ }^{13}$ was of great value. Alignment charts giving particle energy vs magnctic rigidity were essential during the film reading and subsequent calculations. (See Figs.18-22.) The chart for protons is due to. $J$. De Pangher ${ }^{14}$ others were constructed by the author. Stopping Power. The stopping power of the gas mixture in the chamber immediately after expansion was calculated as 0.213 relative to dry air at $760 \mathrm{~mm} \mathrm{Hg}$ and $15^{\circ} \mathrm{C}$. Range-energy curves were prepared for each particle, from the data and techniques of Livingston and Bethe; ${ }^{15}$ Aron, Hoffman, and Williams; ${ }^{16}$ and Bethe. 17 The rangeenergy relations were checked experimentally for protons, tritons, and $\mathrm{He}^{4}$. The energies of a few long tracks ending in the chamber were determined from their magnetic rigidity and their ranges measured with a long flexible ruler. The calculated and measured ranges agreed within 5 to $10 \%$, which is within the experimental error expected. In addition, theoretical track endings were drawn and compared to the experimental track endings obtained; agreement was excellent. 


\section{Errors in Measurement}

Complete analysis of an event requires, for the identification of the particles and subsequent calculations, measurements of radius of curvature, dip angle, beam angle, height of track in chamber at the point of curvature measurement, horizontal distance from point of curvature measurement to vertical axis of chamber, magnetic field strength, and total track length when track ends in the illuminated region of the chamber. The incident neutrons are assumed to enter the chamber in a parallel beam and the ratio of the number of star $s$ observed in the collimated region to the number outside the region verifies this assumption.

Only those events which originated in a predetermined region of the chamber were analyzed. The acceptable region was determined primarily by the dimensions of the final collimator and was of rectangular cross section ( 1 inch high and 3 inches wide) with a length of 12 inches along the beam direction. This region was centered in the chamber so that its upper and lower defining planes were $2-1 / 4$ and $1-1 / 4$ inches from the chamber bottom.

Radius of Curvature. The curvature of a track is measured by reprojecting it life-size on a translucent screen oriented to contain the plane of the track, and then matching it with one of a series of arcs inscribed on a set of lucite templates. (See Fig. 15.) In the range of radii generally encountered the arcs increascd in increments nf $2 \%$ to $4 \%$ between successive curves: It was generally possible to conclude that only one curve was a best fit or, at worst, that the choice lay between two adjacent template arcs. In a number of past experiments experience has shown that the error in curvature measurement amounts to about $0.1 \mathrm{~mm}$ error in the sagitta independent of the particular curvature and track length; for a track of true radius $50 \mathrm{~cm}$, with $20 \mathrm{~cm}$ of track available, this would result in a $1 \%$ error in radius-of-curvature determination. The uncertainties here are therefore assumed to be on the order of $3 \%$. One picture in ten was taken without the magnetic field; from measurements of tracks made under these conditions it was concluded that errors due to turbulence 
were negligible in comparison with the above measurement uncertainties. Dip Angle and Beam Angle. Angular measurements rest on the verified assumption that all neutrons enter the chamber in a parallel beam; the chamber is aligned with the beam axis, vertically and horizontally. The accuracy of reprojection and of angular measurements with the apparatus used in this experiment has been extensively investigated by Powell et al ${ }^{10}$. They concluded that dip angles a could be determined to $\pm 1.5^{\circ}$ in the region $0^{\circ}$ to $50^{\circ}$, and that beam angles $\beta$ could be determined to $\pm 1^{\circ}$. (See Fig. 15 and Appendix I: Definitions.) The latter uncertainty includes the systematic error in aligning the reference cross marks on the top glass of the chamber with the direction of the neutron beam in the reprojection process.

Complete analysis of stars was limited to those events which had all their prongs within dip angles of $\pm 50^{\circ}$. This restriction was necessary because when the prong under consideration is too steep accurate superposition of the two stereoscopic images and accurate measurements of curvature and dip angle are impossible in a great many cases. Events with one or more prongs exceeding the $50^{\circ}$ dipangle restriction were recorded, and identified and analyzed to the extent possible. Geometrical correction factors, discussed in a subsequent section, were applied to each event that satisfied the dipangle limitations, to take care of this imposed "blindness." Magnetic Field. As previously noted, an ammeter in the camera dome indicating the magnet current is photographed simultaneously with the chamber pictures. A larger ammeter on the magnet-control panel also indicates this current, and its readings were recorded by an observer. The field strength is determined from these data and a magnetization curve. The magnet field varies by $6 \%$ over the region where tracks were measured, and an accurate map of the field is used to determine the field strength at the center of the measured part of the track. Since the field varies quite slowly over the useful region of the chamber, second-order corrections were not necessary.

The compounded errors of measurement of dip angle, curvature, and field strength enter into the calculated energy of the particle. 
The probable error in energy determination is estimated as $5 \%$ 。

\section{Calculations}

The analysis of an event is completed by making appropriate calculations for the particle energy, azimuthal angle $\phi$, scatter or beam angle $\theta$, and the energy (in some cases minimum only) of the incident neutron; for elastic scattering the scatter angle of the incident neutron in the center-of-mass system is also required. All calculations were made relativistically by use of formulas developed in Appendix II. As has already been indicated in Identification Procedure (III, 2), not all two-pronged events were unambiguously identified during the film reading process, so that calculations under two assumptions as to particle identity were desirable; for one-pronged stars it was desirable to calculate each event both as $\angle \mathrm{He}^{4} 7$ and as $\angle \mathrm{He} 3$. . A standard for mula and uniform procedure for calculation, suitable to a. CRC 102-A computer, were devised for computing the momentum and energy of the secondary neutron(s) and the incident neutron. This procedure was applicable to all types of events except $\angle \mathrm{DT}$ ) which (as is shown below) is very easily calculated. Inputs were the energies and momentum components of the observed charged particles and the binding energies for the several reaction types. From the standpoint of simplicity of programming and over-all computer time it turned out that the simplest approach was to calculate each two-pronged event for every possibility except $\angle \mathrm{DT}\rangle$, i.e., as $\langle\mathrm{P}$ T or $\mathrm{TP}\rangle ; \angle \mathrm{PD}$ or $\mathrm{DP}\rangle, \angle \mathrm{DD}\rangle$, and $[\mathrm{PP}]$, as well as computing each one-pronged event both as $\left[\mathrm{He}^{4}\right.$ and as $\left\langle\mathrm{He}^{3}\right]$. Sample calculation sheets, showing input data for the computer and computer outputs (indicated by arrows) are included in Appendix II. Calculations for the computer inputs were done with a desk calculator. Charged-particle scatter and azimuthal angles were similarly:determined; charged-particle energies were determined with alignment charts. Center-of-mass-system scatter angle for the incident neutron in $\left\lfloor\mathrm{He}^{4}\right]$ was by nomogram for the formula given in Appendix II. 
Two-Pronged Stars. A total of 416 two-pronged stars was observed-293 that met the dip-angle limitations and 123. that did not. Of the 293 meeting dip-angle requirements only 178 turned out, on calculation, to have been induced by an incident neutron of energy equal to or greater than $160 \mathrm{Mev}$. Of the 123 not meeting dip-angle requirements calculations were possible for 94, and of these only 48 turned out to have been induced by an incident neutron of energy equal to or greater than $160 \mathrm{Mev}$; energy calculations in this group are not so reliable as those in the first. This breakdown is summarized in Table I.

\section{Table I}

\section{Breakdown of calculations}

for 416 two-pronged stars satisfying dip-angle requirements

Incident-neutron energy

Greater than $160 \mathrm{Mev}$

Less than $160 \mathrm{Mev}$

Undetermined
Dip angle

\begin{tabular}{|c|c|c|}
\hline $\begin{array}{c}\text { Less } \\
\text { than } \\
50^{\circ}\end{array}$ & $\begin{array}{c}\text { Greater } \\
\text { than } \\
50^{\circ}\end{array}$ & All \\
\hline 178 & 48 & 226 \\
\hline \multirow[t]{2}{*}{115} & 46 & 161 \\
\hline & 29 & 29 \\
\hline 293 & 123 & 416 \\
\hline
\end{tabular}

The $\angle D T 7$ process, being a two-body problem, is simply calculated. The deuteron and triton, both of which form visible tracks in the chamber, account for all the nucleons involved in the reaction. The energy of the incident neutron can be calculated as the sum of the charged-particle energies plus the binding energy of the reaction; it can also be independently calculated from the sum of the beam components of momentum of the deuteron and triton. The energies computed in these two ways should be equal within experimental error. Further checks are available: the transverse momenta of the deuteron and triton must be equal and opposite; the deuteron and triton must be separated by $180^{\circ}$ in azimuthal angle. There was excellent conformance 
with these requirements in the few $\langle D T\rangle$ cases observed.

In the $\angle P T\rangle$ and $\angle D D\rangle$ processes the two charged particles whose tracks are visible in the chamber account for only four of the five nucleons involved in the reaction; the path of the fifth particle, a neutron, is invisible: Since the direction of the incident neutron is known, only its energy and the beam and transverse-momentum components of the ejected neutron are undetermined. Available conservation-of-energy and-momentum relations are just sufficient to permit solution of the problem.

In the $\angle P D\rangle$ process two neutrons are ejected and there are too many unknowns to permit solution of the problem. By considering the two ejected neutrons as a single lumped particle of two neutron masses, and of momentum just sufficient to balance the event, an "iucidentneutron energy".. can be calculated. The energy so determined is only a minimum energy, for the incident neutron, but it was calculated for the $\langle\mathrm{PD}\rangle$ events.

The $[\mathrm{PT}]$ process, in which three neutrons are ejected, is also indeterminate. A minimum energy for the incident neutron was calculated by considering the three neutrons as a single lumped particle. One-Pronged Stars. A total of 570 single tracks at least $2 \mathrm{~cm}$ long was observed --449 that met the dip-angle limitations and 121 that did not. For the 449 meeting dip-angle requirements, calculations 'were possible for 446 and of these only 66 turned out to have been induced by an inctienl ueution of cncrgy at least lifn Mev. For the 121 not meeting dip-angle requirements calculations were possible for 108 and of these only 34 turned out to have. been induced by an incident neutron of energy at least $160 \mathrm{Mev}$; energy calculations in this group are not so reliable as those in the first. This breakdown is summarized in Table II. 
for 570 one-pronged stars satisfying dip-angle requirements

Incident neutron energy

Greater than $160 \mathrm{Mev}$

Less than $160 \mathrm{Mev}$

Undetermined
Dip angle

\begin{tabular}{|c|c|c|}
\hline $\begin{array}{c}\text { Less } \\
\text { than } \\
50^{\circ}\end{array}$ & $\begin{array}{c}\text { Greater } \\
\text { than } \\
50^{\circ} \\
\end{array}$ & All \\
\hline 66 & 34 & 100 \\
\hline 380 & 74 & 454 \\
\hline 3 & 13 & 16 \\
\hline 449 & 121 & 570 \\
\hline
\end{tabular}

In addition, a total of 525 single tracks less than $2 \mathrm{~cm}$ long was observed. These tracks were simply recorded, and no measurements or calculations were attempted.

The $\left.\angle \mathrm{He}^{4}\right]$ elastic-scattering interaction can be simply calculated. A unique relativistically correct formula relates the incident-neutron energy to the observed energy and scattering angle of the recoil $\mathrm{He}^{4}$ in the laboratory system. This formula is given in Appendix II and, although it is rather complex, is readily solved by a nomogram. Such a nomogram was constructed, but used only to check the solutions of the automatic computer, which used the uniform procedure already mentioned. The scatter angle $\theta^{\prime}$ of the neutron in the center-of-mass system is given in terms of the recoil angle $\theta$ of the $\mathrm{He}^{4}$ in the laboratory system by

$$
\tan \theta=\sqrt{1-\beta^{2}} \cot \frac{\theta^{8}}{2}
$$

where $\beta$ is for the velocity of the center-of-mass system relative to the laboratory system. This formula was solved by a simple circular nomogram. 
The $\left[\mathrm{He}^{3} 7\right.$ process, in which two neutrons are ejected, is indeterminate. A minimum energy for the incident neutron was calculated by considering the two neutrons as a single lumped particle.

The formulas used in the above calculations, and the derivations of some, are given in Appendix II.

\section{CORRECTIONS}

Because of the restriction of $\pm 50^{\circ}$ in dip angle in the film-reading process a geometrical correction factor must be applied to each meas ured event to determine the number of events that would have been observed without the restriction. For single tracks the correction factor is a function only of the beam angle $\theta$ of the observed particle; for twopronged events the beam angles of both particles, and their difference in azimuth, are involved.

It has been assumed that all processes in this experiment occur with azimuthal symmetry and, as is shown in a subsequent section, the experimental data verify this assumption. For any given type of event, however, there is a range of azimuthal angles for which the" corresponding dip angles exceed the arbitrarily imposed maximum dip angle. A corresponding number of events of this type, together with its associated properties (particle energies, scatter angles, etc,) are thus "lost". The geometrical correction factors are designed to recover these data.

\section{Single-Pronged Stars}

Consider an $\left[\mathrm{He}^{4}\right]$ event in which the recoil helium nucleus has a scatter angle $\theta$. From the isotropic distribution in azimuthal angle $\Phi$. we include in the measured data only those whose dip angle a given by

$$
a \cdot=\sin ^{-1}(\sin \theta \sin \phi)
$$

is equal to or less than some maximum, say $a_{0} \cdot$. Now, there is some value $\phi_{0}$ of $\phi^{\prime}$, less than $90^{\circ}$, for which one has 


$$
\sin ^{-1}\left(\sin \theta \sin \phi_{0}\right)=a_{0} .
$$

Between $\phi_{0}$ and $90^{\circ}$ the dip angle would exceed $\alpha_{0}$. In the first quadrant, therefore, only $\phi_{0}{ }^{\circ}$ out of $90^{\circ}$ yield measured data. The situation is repeated in the remaining three quadrants, so that we find this type of event in just $4 \phi_{0}^{\circ}$ out of $360^{\circ}$. Thus, if $N$ events of this type we re obse rved in $4 \phi_{0}{ }^{\circ}$, we should have observed $N \times \frac{360}{4 \phi_{0}}$ such events without the restriction in dip angle. The geometrical correction, or weighting factor, for this type of event is therefore $90 / \phi_{0}$, whe re $\phi_{0}=\sin ^{-1}\left(\sin a_{0} / \sin \theta\right)$. In Fig. 16 thio correctiun factor has been plotted as a function of $\theta$ for $a_{0}=50^{\circ}$; its maximum value, which occurs at $\theta=90^{\circ}$, is seen to be 1.80 .

\section{Two-Pronged Stars.}

Consider a $[\mathrm{PT}]$ event in which the proton has scatter angle. $\theta_{1}$, the triton has scatter angle $\theta_{2}$, and the two tracks are separated in azimuth by an amount $\Delta \phi$. From the isotropic distribution we include in the measured data only those whose dip angles, given by

$$
\begin{aligned}
& a_{1}=\sin ^{-1}\left(\sin \theta_{1} \sin \phi_{1}\right), \\
& a_{2}=\sin ^{-1}\left(\sin \theta_{2} \sin \phi_{2}\right)
\end{aligned}
$$

are simultaneously equal to or less than some maximum, say $a_{0}$. To determine the correction factor we need to find the azimuthal angular intervals that would satisfy the imposed condition for both the dip angles, with the added condition that $\phi_{1}$ and $\phi_{2}$ differ at all times by $\Delta \phi$. This was done graphically by means of Fig. 17, which is a plot of the relation

$$
\phi=\sin ^{-1}\left( \pm \sin 50^{\circ} / \sin \theta\right)
$$

as $\theta$ varies from $50^{\circ}$ to $130^{\circ}$.

The procedure was as follows: a transparent plastic triangle was laid over the figure; an ink dot was placed on the triangle at $\theta=\theta_{1}$ and $\phi=0$ and a second dot placed at $\theta=\theta_{2}$, and $\phi=\Delta \phi$; the 
triangle was then moved vertically through the plot, with the dots always on the ordinates at $\theta_{1}$ and $\theta_{2}$ respectively, so that the $\theta_{1}$ dot moved from $\phi=0^{\circ}$ to $\phi=360^{\circ}$. So long as both dots a re outside the ovals the conditions for including the event are satisfied. Consequently, if both dots are outside the ovals for $\Delta \phi_{0}{ }^{\circ}$ during the sweep, the geometrical correction, or weighting factor, for this type event is simply $360 / \Delta \phi_{0}$. It will be noted that the scale in $\theta$ is not complete; if either particle has a scatter angle equal to or less than $50^{\circ}$ or equal to or greater than $130^{\circ}$, its corresponding dot would never enter an oval and the correction is more simply obtained from Fig. 16, based only on the second track; if the scatte $r$ angles of both the particles are in the intervals just defined, the weighting factor is unity. In practice lusst of the weighting factors we re obtainable from Fig. 16, and ranged from unity to 1.8 ; a few re quired the use of Fig. 17, and were generally between 1.8 and 2.5 , and one went as high as 3.2 . 


\section{RESULTS AND DISCUSSION}

\section{Inelastic Events}

Table III summarizes the results for those inelastic events which, having dip angles equal to or less than $\pm 50^{\circ}$, were subject to a detailed analysis and which also satisfied the condition that they were induced by an incident neutron whose energy was at least $160 \mathrm{Mev}$.

Table III

Summary of analysis for inelastic events of dip angle $\leqslant \pm 50^{\circ}$

\begin{tabular}{|c|c|c|c|c|}
\hline Reaction & & $\begin{array}{l}\text { Actual } \\
\text { number } \\
\text { measured }\end{array}$ & $\begin{array}{l}\text { Weighted } \\
\text { number }\end{array}$ & $\begin{array}{l}\text { Standard } \\
\text { deviation } \\
\text { (percent) }\end{array}$ \\
\hline$\lfloor\mathrm{PT}\rangle$ & & 103 & 150.1 & 9.9 \\
\hline$\langle\mathrm{DT}\rangle$ & & 3 & 4.3 & 57.8 \\
\hline$\angle P D\rangle$ & & 46 & 67.5 & 14.7 \\
\hline$\angle \mathrm{DD}]$ & & 18 & 29.9 & 23.6 \\
\hline$\langle\mathrm{PP}\rangle$ & & 8 & 12.3 & 35.4 \\
\hline$\angle \mathrm{He}^{3} /$ & & 6 & 9.5 & 40.8 \\
\hline & Total & 184 & 273.6 & 7.4 \\
\hline
\end{tabular}

The weighted numbers are the total numbers of events of each type that would have been observed if the dip angle had not been restricted; they were determined by application, to each observed event, of the previously described geometrical correction factors. The deviations are statistical standard deviations based on the actual number of events measured as given in the second column. 
As has been indicated in Table I, 48 two-pronged events were identified which met the requirement placed on incident-neutron energy but did not satisfy the dip-angle limitation; in addition the re were 29 incompletely identified events (incident-neutron energy the refore indeterminate) which did not satisfy the dip-angle limitation. The former had a distribution among the several types of two-pronged events closely approximating that indicated in Table III; the latter can be arranged so as to give the same distribution. The distribution is indicated in Table IV.

\section{Table T.V}

Distribution of identified two-pronged events

Dip-angle criterion

\begin{tabular}{|c|c|c|c|c|}
\hline \multicolumn{5}{|c|}{ Reaction } \\
\hline $\begin{array}{c}\angle \mathrm{PT}\rangle \\
(\%)\end{array}$ & $\underset{(\%)}{\operatorname{LDT} 7}$ & $\begin{array}{l}\langle\mathrm{PD}\rangle \\
(\%)\end{array}$ & $\langle\underset{(\%)}{\langle\mathrm{DD}}\rangle$ & $\underset{(\%)}{\langle\mathrm{PP}}$ \\
\hline 57.9 & 1.7 & 25.8 & 10.1 & 4.5 \\
\hline 56.8 & 1.6 & 25.6 & 11.3 & 4.7 \\
\hline 56.3 & 0.0 & 25.0 & 12.5 & 6.2 \\
\hline
\end{tabular}

The 29 incompletely identified events were distributed as 18 /P?], $5 / \mathrm{D} ? 7$ and $6 / \mathrm{T} ? 7$. Table I indicates that not less than half of them should be attributed to incident neutrons having energy of at least $160 \mathrm{Mev}$; their distribution is such that they could easily be arranged to conform to that in Table IV.

Discussion of Geometrical Correction. The difference between Columns 3 and 2 of Table III indicates that (exclusive of $\left[\mathrm{He}^{3}\right]$ reactions) the geometric corrections have predicted the occurrence. of 86 two-pronged events in the regions rejected by the dip-angle limitation. In comparison, 48 completely identified events satisfying the incident-n eutron energy requirement and 29 incompletely identified events of unknown neutron 
energy were actually observed. Reference to the third column of Table I reveals that $51 \%$, or about 15 , of the latter 29 events should be attributed to neutrons of at least $160 \mathrm{Mev}$; these, with the 48 identified events, total 63 where 86 were expected. However, it has been previously indicated that energy calculations for events that exceeded the dip-angle limitations were not particularly reliable; for the larger number of actual events that did not exceed the dip-angle requirement the second column of Table I indicates that $61 \%$ were due to neutrons of at least $160 \mathrm{Mev}$, and if this be applied to the 123 events that comprise the third column of Table I there result 75 where 86 are expected. It is concluded that, within the statistical errors of Table III, the 63 to 75 events actually observed in the excluded regions is compatible with the 86 predicted by the geometrical corrections.

The validity of the geometric correction applied to the singlepronged inelastic events, $\left[\mathrm{He}^{3} /\right.$, is discussed in the following section.

\section{Elastic Events}

Table $\mathrm{V}$ summarizes the results for those single-pronged events, primarily elastic, which, having dip angles equal to or less than $\pm 50^{\circ}$, were subject to a detailed analysis and which also satisfied the condition that they were induced by an incident neutron whose energy was at least $160 \mathrm{Mev}$.

Table V

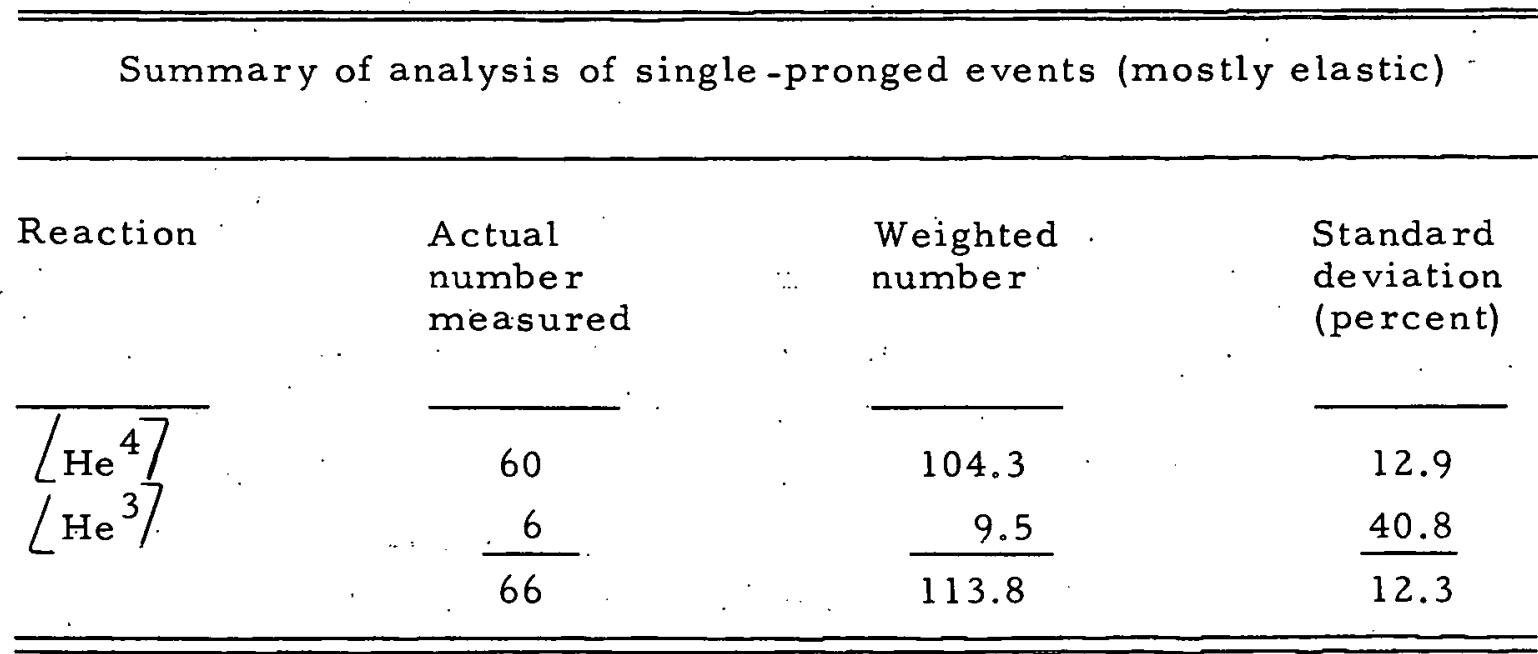


As before, the weighted numbers are the total numbers of each type that would have been observed if the dip angle had not been restricted. Standard deviations are based on the actual number of events measured as given in the second column.

As has been indicated in Table II, 34 single-pronged events we re identified which met the incident-neutron energy requirement but did not satisfy the dip-angle limitation; in addition there were 13 unresolved events (incidenl-ueutron crorgy therefore not determined) which did not satisfy the dip-angle limitation. The former were resolved as $32 / \mathrm{He}^{4} /$ and $2\left[\mathrm{He}^{3} /\right.$, which is not incompatible with the relative numbers of these events shuwu in the cecond collumn of Table V. Finally, 525 single tracks less than $2 \mathrm{~cm}$ long we re observed.

Discussion of Missing Tracks. The angular distribution of the elastically scattered neutrons, in the center-of-mass system, shows a lack of neutrons in the forward direction; this is due to the short range of the recoils. (See Fig. 9.) Presumably these missing recoils are among the 525 tracks that were too short to measure'. The experimental points of Fig. 9, which are relative values of $d \sigma / d \Omega$ as a function of the neutron scatter angle $\theta$, have been fitted by the smooth gaussian drawn thereon. In Fig. 10 the 104.3 weighted $\left[\mathrm{He} \mathrm{H}^{4} /\right.$ events of Table V are plotted to show the number of neutrons scallered per $10^{\circ}$ interval in the renter-of-mass system. The experimental points of Fig. 10 are relative values of $(d \sigma / d \Omega) \sin \theta$; the curve is the corresponding function for the gaussian previously fitted to the data of Fig. 9. The experimental weighted events show 97.1 neutrons scattered at angles greater than $10^{\circ}$. When the area under the curve from $10^{\circ}$ to about $57^{\circ}$ is normalized to this number it is found that the area from $0^{\circ}$ to $10^{\circ}$ corresponds to 18.9 weighted events. Experimentally, only 7.2 weighted events were observed for the $0^{\circ}$ - to $-10^{\circ}$ interval. Thus approximately 11.2 weighted events are missing and should be among the previously mentioned very short tracks.

Examination of the second column of Table II shows that of the 449 single-pronged events whose associated neutron-energy determinations were the most reliable, 66 or about $15 \%$ were due to neutrons 
of energy at least $160 \mathrm{Mev}$. If this ratio applied to the 525 very short tracks, then about 79 are available to account for the missing tracks. This is far more than is required; it is probable that a much smaller proportion of the very short tracks was actually induced by beam neutrons of at least $160 \mathrm{Mev}$.

In view of the above, the cross-section calculations in a subsequent section are based on the "corrected weighted total" of $97.1+$ 18.9, or 116 elastic $/ \mathrm{He}^{4} /$ events.

Discussion of Geometrical Correction. The difference between Columns 3 and 2 of $\mathrm{T}$ able $\mathrm{V}$ indicates that the geometric corrections have predicted the occurrence of 48 single-pronged events in the regions rejected by the dip-angle limitations. In comparison, 34 completely identified events satisfying the inçident-neutron energy requirement, 13 unresolved events of unknown neutron energy, and 525 very short tracks of unknown angle and neutron energy were actually observed. If the 34 identified events be accepted as actually due to neutrons of energy greater than $160 \mathrm{Mev}$ it can be shown that 4 of the 13 unresolved events and 16 of the 525 short tracks belong to the rejected regions and have the minimum required incident-neutron energy. These total 54, in good agreement with the 48 predicted by the geometrical corrections. However, while reasonably confident of the identification of the 34 events, the author is much less confident that they were all induced by neutrons of ene rgy of at least $160 \mathrm{Mev}$. It has been previously indicated that energy calculations for events that exceeded the dip-angle limitation were not particularly reliable. If one compares the data in Columns 2 and 3 of Table II it is seen that the ratios therein of numbers of events due to neutrons of energy greater than $160 \mathrm{Mev}$ to those due to neutrons of energy less than $160 \mathrm{Mev}$ are widely divergent. The proportions of Column 2 are more reliable, and if they are applied to the 121 events which comprise Column 3 only 1.8 would be due to neutrons of the proper energy. Again it can be shown that 16 of the 525 short tracks (used above, and "correctly" derived in the first place) belong to the rejected regions 
with the minimum required neutron energy. We thus arrive at $18+16$, or only 34 where 48 we re expected. It must the refore be concluded that something. like 34 to 54 are found, with emphasis on the lower figure, where 48 are predicted, and that this is only marginal agreement within the statistical errors of Table V.

\section{Meson Events}

A careful search was made for meson-producing events. Three instances of negative-pion production in helium were observed, one by each of the reactions $\mathrm{He}^{4}\left(\mathrm{n}, \mathrm{pn} \pi^{-}\right) \mathrm{He}^{3}, \mathrm{He}^{4}\left(\mathrm{n}, \mathrm{d} \pi^{-}\right) \mathrm{He}^{3}$, and $\mathrm{He}^{4}\left(\mathrm{n}, \mathrm{dp} \pi^{-}\right) \mathrm{d}$. One possible case of posilive-pion production was notcd. These events have not been weighted because no restriction on dip angle was imposed. Since the thresholds for the reactions are approximately $200 \mathrm{Mev},{ }^{5}$ it is clear that they were induced by incident neutrons of energy greater than $160 \mathrm{Mev}$.

\section{Cross Sections}

In order to obtain absolute cross sections, the total number of weighted events, exclusive of pion events, comprised of 273.6 inelastic and 116 elastic events, has been normalized to an interpolated $1-\mathrm{He}^{4}$ total cross section of 100 millibarns at $300 \mathrm{Mev}$. A standard deviation of $10 \%$ is estimated for this value. The interpolated total cross section was based on plots of $\sigma_{t}$ vs $A^{2 / 3}$, the data used were those of Hillman et al. ${ }^{4}$ for $\mathrm{H}, \mathrm{He}, \mathrm{C}, \mathrm{N}$, and $\mathrm{O}$ at $88 \mathrm{Mev}$; those of $\mathrm{T}$ aylor ${ }^{18}$ for $\mathrm{H}, \mathrm{D}, \mathrm{C}$, and $\mathrm{O}$ at $169 \mathrm{Mev}$; those of DeJuren, ${ }^{19}$ Fox et al. , 20 and Nedzel ${ }^{2 l}$ for $\mathrm{H}, \mathrm{D}, \mathrm{Be}, \mathrm{C}$, and $\mathrm{O}$, at $270 \mathrm{Mev}, 280 \mathrm{Mev}$, and $410 \mathrm{Mev}$ respectively.

The results are listed in Table VI. 
Table VI

\begin{tabular}{|c|c|}
\hline \multicolumn{2}{|c|}{ Cross sections for inelastic and elastic events } \\
\hline $\begin{array}{l}\text { Reaction } \\
\text { Inelastic }\end{array}$ & $\begin{array}{l}\text { Cross section } \\
\quad(\mathrm{mb})\end{array}$ \\
\hline $\mathrm{He}^{4} \cdot(\mathrm{n}, \mathrm{pn}) \mathrm{t}$ & $38.5 \pm 3.8$ \\
\hline $\mathrm{He}^{4}(\mathrm{n}, \mathrm{d}) \mathrm{t}$ & $1.1 \pm 0.6$ \\
\hline $\mathrm{He}^{4}(\mathrm{n}, 2 \mathrm{np}) \mathrm{d}$ & $17.3 \pm 2.6$ \\
\hline $\mathrm{He}^{4}(\mathrm{n}, \mathrm{dn}) \mathrm{d}$ & $7.7 \pm 1.8$ \\
\hline $\mathrm{He}^{4}(\mathrm{n}, 2 \mathrm{n} 2 \mathrm{p}) \mathrm{n}$ & $3.2 \pm 1.1$ \\
\hline $\mathrm{He}^{4}(n, 2 n) \mathrm{He}^{3}$ & $2.4 \pm 1.0$ \\
\hline \multicolumn{2}{|l|}{ Elastic } \\
\hline $\mathrm{He}^{4}(n, n) \mathrm{He}^{4}$ & $29.8 \pm 3.8$ \\
\hline$\underline{\sigma_{\text {inel }}}=0.70 \pm 0.10$ & $\underline{\sigma_{\mathrm{el}}}=0.30 \pm 0.06$ \\
\hline$\sigma_{\mathrm{t}}$ & $\sigma_{\mathrm{t}}$ \\
\hline
\end{tabular}

The cross section for negative-pion production: is, from the few events observed; $0.8 \pm 0.4$ millibarn. . 
5. Errors

Measurement errors and the uncertainties encountered in the identification procedures have been discussed in. Chapter III, Method of Analysis of Events. The probable error in energy determinations has been estimated as about $5 \%$; this applies to observed particles for dip angles within the $50^{\circ}$ limitation. Derived energies for incident neutrons are estimated to have an average probable error of about $10 \%$, but those determinations involving large angles in elastic scattering were less reliable.

In the determination of cross. sections and various angular and energy distributions the chief solirse of error is statistical. Only 244 acceptable events of all kinds were observed within the energy and angular limitations; although these were geometrically corrected to yield a weighted total of 390 , the statistics, are tied to the lower figure and its subdivisions and are not improved. Consequently, all other errors are considered negligible in comparison with the statistical errors.

In view of the above, the errors already quoted in Tables III, V, and VI, as well as those indicated in the several angular and energy distributions (Figs. 1-12), a re statistical standard deviations based only on the number of events actually analyzed within the energy and angular limitations.

\section{Comparison with Theory}

The neutron helium scattering problem, for monoenergetic neutrons of $90 \mathrm{Mev}$ and $200 \mathrm{Mev}$, has been examined by Heidmann, 6, 7 using the Born approximation and gaussian potentials and wave functions. Heidmann's theoretical relative cross sections are compared with the experimental findings of the study reported here in $: . .$. Table VII. No theoretical estimates are available for 300 -Mev neutrons, and it should be noted that the neutron beame of this experiment exhibits a rather wide energy spectrum (Fig. 13) and further that the results are for neutrons of energy equal to or greater than $160 \mathrm{Mev}$. For a somewhat more realistic comparison, Heidmann's $90-\mathrm{Mev}$ estimates are normalized to a

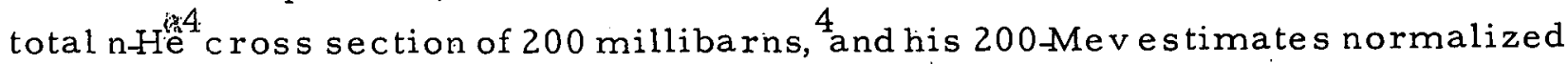


to a total cross section of 110 millibarns; the latter cross section is an interpolated estimate employing the data and procedure outlined in Section 4, Cross Sections (above).

Table V.II

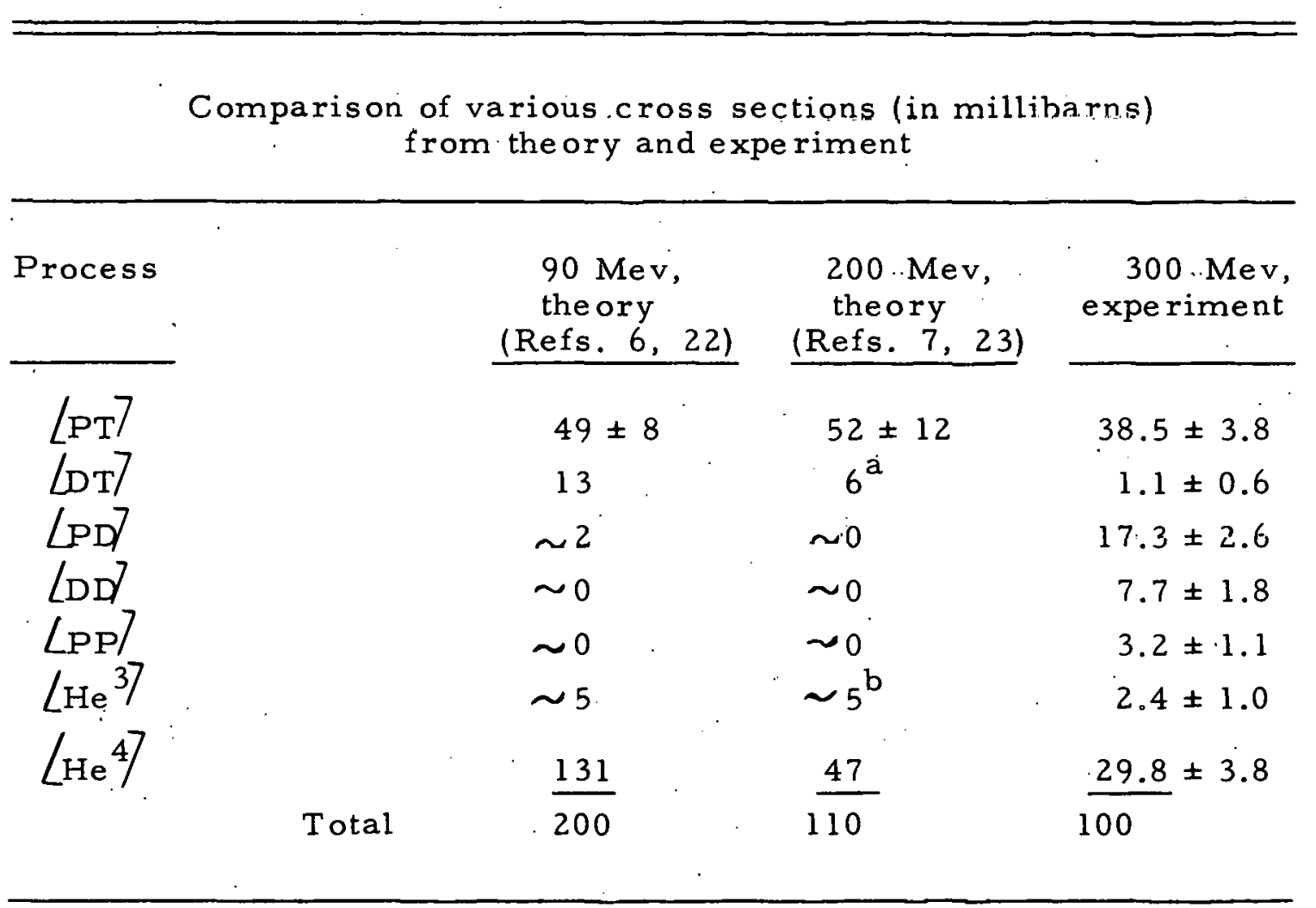

${ }^{a}$ Some liberties have been taken with Heidmann's estimates. His result for $[\mathrm{DT} /$ was $1 / 10 \mathrm{mb}$, with the statement that it was approximately two orders of magnitude too small.

${ }^{b}$ Heidmann made no estimate for $\left[\mathrm{He}^{3}\right]$ at $200 \mathrm{Mev}$. This value is obtained by taking $1 / 10$ of his /PT] value, following his method of estimating this cross section at $90 \mathrm{Mev}$. 
On consideration, first; of the gross features of this table it is seen that the theoretical predictions of Heidmann are in good agreement with the experimental observations of DeJuren and Moyer that total cross sections drop rapidly with energy above about $100 \mathrm{Mev}$ and that this drop is primarily due to a decrease in the elastic part of the cross section. 24,25 The theoretical ratio of the elastic to the total cross section is" about 0.65 at $90 \mathrm{Mev}$ and about 0.43 at $200 \mathrm{Mev}$. Experimentally, Tannenwąld observed a ratio of about 0.51 at $90 \mathrm{Mev}^{1}$ (actually neutrons of ene rgy greater than $40 \mathrm{Mev}$ in a sjectrum peaked at about $75 \mathrm{Mev}$, and extending from about 40 to $115 \mathrm{Mev}) ;$ Swartz observed a ratio of about 0.43 at $200 \mathrm{Mev}^{3}$ (actually neutrons of energy greater than 50 Mev. in a spectrum peaked at about $195 \mathrm{Mev}$, and extending from about 50 to 230 Mev); this experiment yields a ratio of about 0.30 at $300 \mathrm{Mev}$ (actually neutrons of energy greater than $160 \mathrm{Mev}$ in a spectrum peaked at about $310 \mathrm{Mev}$, and extending from about 160 to $340 \mathrm{Mev}$ ).

The theory predicts [PT] as.the dominant process at both 90 Mev and 200 Mev, with relatively small variation of the cross section with energy; at $90 \mathrm{Mev}$ the [PT/, cross section is about $37 \%$ of the elastic cross section designated $\left[\mathrm{He}^{4}\right]$, but at $200 \mathrm{Mev}$, because of the rapid drop in the elastic cross section, this ratio is about 1.1 . This experiment shows that $[\mathrm{PT}]$ continues to be the dominaul process and varies perháps a little more strongly with energy; the ratio of the [P.T] cross section to the elastic cross section is about 1.5 , since the elastic cross section has decreased more rapidly than that of [PT].

The theoretical cross section for LDT/ decreases substantially with energy from $90 \mathrm{Mev}$ to $200 \mathrm{Mev}$. This is in agreement with the expected energy dependence of the direct pickup process; 26 theoretical considerations $^{27}$ and experiment ${ }^{28}$ reveal a rapid decrease, with increas ing energy, of the cross section for the formation of pickup deuterons by the direct pickup process. At $90 \mathrm{Mev}$ Tannenwald observed that pickup deuterons made a substantial contribution to the $\angle \mathrm{DT} /$ cross section, while at $200 \mathrm{Mev}$ Swartz concluded that the [DT/ process seemed to have a very low probability. This experiment is in agreement with the theoretically predicted rapid decrease in the cross section for the LDT] 
process; only three (unweighted) cases were observed within the energy and angular limitations.

Comparison of the theoretical predictions for $\angle \mathrm{PD}\rangle$. and $\angle \mathrm{DD}\rangle$ at 90 and $200 \mathrm{Mev}$ with the experimental results at $300 \mathrm{Mev}$ reveals that Heidmann's analytical procedure probably underestimates the frequency of these reactions. Tannenwald also found these processes to occur with significant frequency at $90 \mathrm{Mev}$.

For $[P P]$ the theoretical predictions at the lower.energies are in agreement with the results at $300 \mathrm{Mev}$; the complete disintegration of helium is relatively infrequent, even at the higher energies; Tannenwald obtained a similar result at $90 \mathrm{Mev}$.

The theoretical predictions for $\left[\mathrm{He}^{3}\right]$ at the lower energies are also in agreement with the $300-\mathrm{Mev}$ results. As indicated in the footnote, Heidmann made no estimate for $\left[\mathrm{He}^{3} /\right.$ at $200 \mathrm{Mev}$ and the value in the table was obtained by using the procedure employed by him for the $90-\mathrm{Mev}$ calculations. In those calculations Heidmann considered $/ \mathrm{PT}\rangle$ and $[\mathrm{He} 37$ as similar processes of the first order and estimated the ratio of $\left[\mathrm{He}^{3}\right]$ to $\langle\mathrm{PT}]$ as

$$
[1 / 4 /(1 / 4+3 / 4)] \cdot\left(V_{\text {Singlet }} / V_{\text {Triplet }}\right)^{2},
$$

or about one-tenth. The experimental ratio at $300 \mathrm{Mev}$ is about 1 to 16 .

For the elastic-scattering case, $\left[\mathrm{He}^{4}\right]$, the theory predicts a significant decrease in cross section with increasing energy; the experimental results are in agreement.

Further comparisons of theory with experiment are found in Section 8, Energy and Angular Distributions (below).

\section{Comparison with Other Experiments}

Similar experiments have been conducted by Tannenwald at 90 $\mathrm{Mev}^{\mathrm{l}}$ and Swartz at $200 \mathrm{Mev;}{ }^{3}$ Tannenwald's experiment was conducted with the same apparatus as described in this paper; the experiment by Swartz was also conducted with a cloud chamber, but analysis was difficult because no -magnetic field was availabie. 
Moulthrop, using a high-pressure diffusion cloud chamber in the magnetic field described herein, studied negative-pion production in the bombardment of helium by $300-\mathrm{Mev}$ neutrons. ${ }^{5}$ The results of Tannenwald and Swartz are compared with the present experimental findings in Table VIII; the results of Tannenwald and Moulthrop are compared with those of this experiment in Table IX, following the analogy originally employed by Moulthrop. ${ }^{5}$ The experimental results at $90 \mathrm{Mev}$ and 200 . Mev have been normalized to total. $\mathrm{n}-\mathrm{He}^{4}$. cross sections of 200 millibarns and 110 millibarns, respectively, as described in Section 6, Comparison with Theory (above).

Table VIII

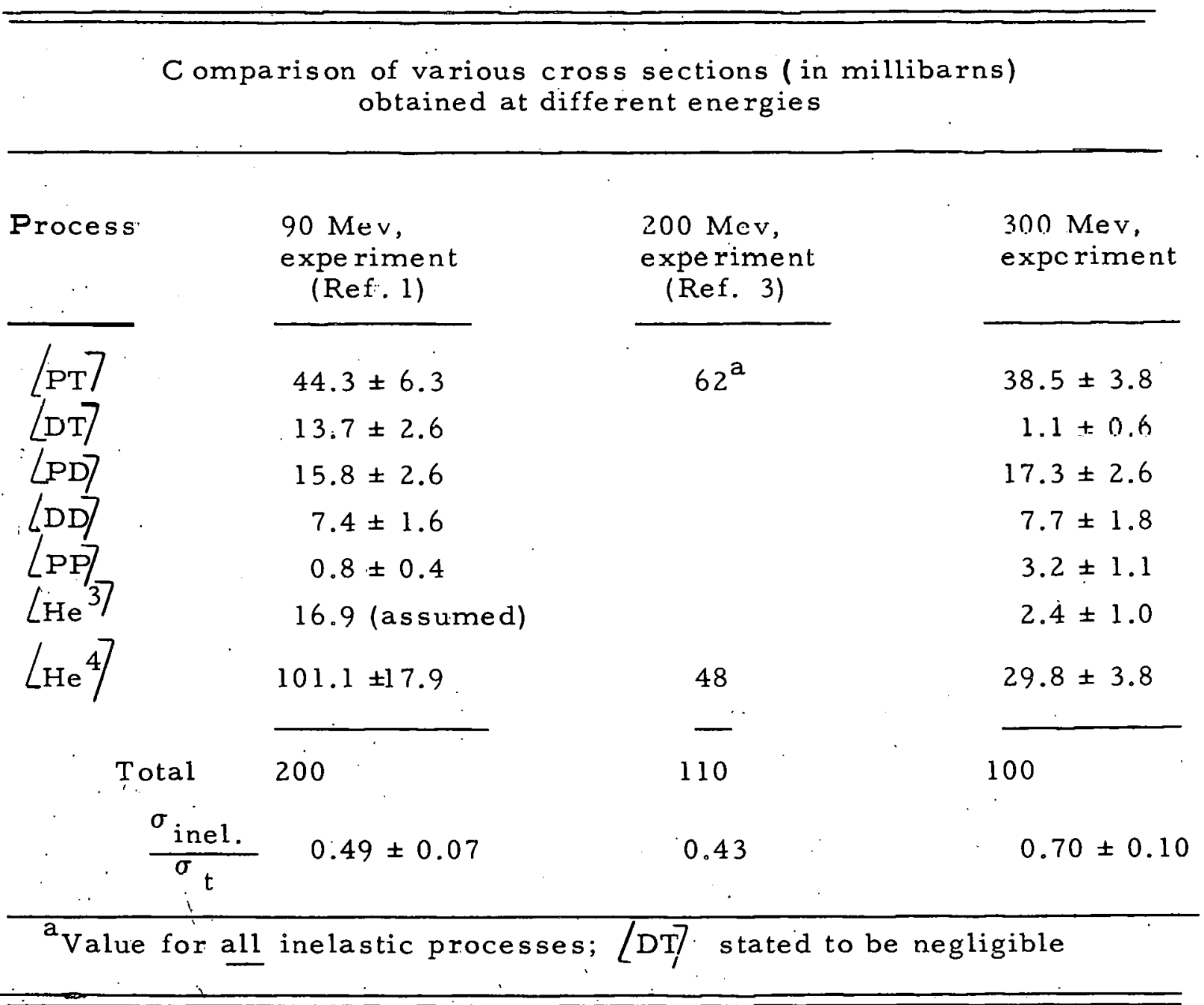


These experimental data illustrate, and are in good agreement with, the earlier observations that total cross sections drop rapidly with energy above about $100, \mathrm{Mev}$ and that this drop is due primarily to the decrease in the elastic part of the cross section. The $90-\mathrm{Mev}$, $200-\mathrm{Mev}$, and 300-Mev total cross sections are 200,110, and $100 \mathrm{milli}$ barns, respectively, while the corresponding elastic cross sections are 101,48 , and 30 millibarns. The corresponding inelastic cross sections reveal significantly smaller energy dependence, particularly between 90 Mev and $200 \mathrm{Mev}$. In view of the relative uncertainties involved, Swartzs inelastic cross section is not incompatible: with a steadily decreasing function of energy. As noted below, Tannenwald's inelastic cross section could be a little high, and his elastic cross section correspondingly low, because of his $/ \mathrm{He}^{3} /$ assumption.

The $90-\mathrm{Mev}$ and $300-\mathrm{Mev}$ data agree that $[\mathrm{PT} /$ is the dominant inelastic process; the decrease of about $13 \%$ with energy is not great. There is also agreement that $\angle P D /$ is next most frequent in occurrence; the increase of about $1,1 \%$ is not great. At $90 \mathrm{Mev}, / \mathrm{DT} /$ is third most frequent in occurrence, 'while at $300 \mathrm{Mev}$ this process is negligible; the importance of the energy dependence of the direct pickup process in this reaction has already been discussed. The $/ \mathrm{DD} /$ cross section appears to remain constant between $90 \mathrm{Mev}$ and $300 \mathrm{Mev}$. The complete disintegration of helium, $[P P]$, although still relatively insignificant, increases four fold from $90 \mathrm{Mev}$ to $300 \mathrm{Mev}$.

There appears to be significant difference between the $90-\mathrm{Mev}$ and $300-\mathrm{Mev}$ values for $\left[\mathrm{He}^{3} /\right.$. It is possible that the $90-\mathrm{Mev}$ value has been overestimated and that the $300-\mathrm{Mev}$ value is underestimated. The same difficulties we re encountered in both experiments in differentiating the helium isotopes $\mathrm{He}^{3}$ and $\mathrm{He}^{4}$ when their ranges did not end in the chamber. Tannenwald, at $90 \mathrm{Mev}$, estimated the $/ \mathrm{He}^{3} / \mathrm{cross}$ section as about one-third of [PT] plus one-third of that portion of the [DP/ cross section attributable to pickup; Heidmann obtained a ratio of about one-tenth, as described above. Both estimates are based on the same phenomenological equivalence; $[\mathrm{PT}]$ and $\left[\mathrm{He}^{3}\right]$ can be considered as similar processes--in the former, the incident neutron interacts with 
and strips a proton off the helium nucleus, while in the latter it interacts with and strips off a neutron. In this experiment special effort was made to identify $\left[\mathrm{He}^{3} /\right.$ events as described in Chapter III, Method of Analysis of Events. The experimental ratio of the $\left[\mathrm{He}^{3} /\right.$ cross section to the $/ \mathrm{PT} /$ cross section at 300 Mev is 1 to 16; Moulthrop observed a ratio of about 1 to 10 for the similar reactions in his experiment. ${ }^{5}$ These results are in bette $\mathbf{r}$ agreement with Heidmann than with Tannenwald. In the 90-Mev experiment $23 \%$ of the tritons from $[\mathrm{PT} /$ were uliserved to end in the chamber while only $5 \%$ of the $\mathrm{He}^{3 /} \mathrm{s}$. (the total number of $\mathrm{He}^{3 /} \mathrm{s}$ being based on the $\mathrm{He}^{3}$ assumption) were observed to stop. At $300 \mathrm{Mev}$ these percentages were $25 \%$ (based on actual number of eveuls, ur $23 \%$ based on weighted number of events) for tritons and zero for $\mathrm{He}^{3_{1}} \mathrm{~s}$. As mentioned in Chapter III, no $\mathrm{He}^{3}$ which on calculation satisfied the neutron-energy criterion were observed to end in the chamber; a few, associated with neutrons of lower energy, had been so identified; in point of actual numbers, Tannenwald found two definite $\mathrm{He}^{3}$ endings. In view of the foregoing, although it is quite possible that $\left(\mathrm{He}^{3}\right)$ may have been underestimated in this experiment, it is believed that this process has been overestimated at $90 \mathrm{Mev}$. Of the three possible explanations advanced by Tannenwald for the observed great difference between phenomenologically similar processes, it appears that the most likely explanation is his first, viz., the number of $\mathrm{He}^{3,} \mathrm{~s}$ was overestimated in the $\mathrm{He}^{3}$ assumption.

As has already been mentioned, the behavior of the elastic.cross section is as expected. In view of the above discussion, the elasticcross-section value at $90^{\circ} \mathrm{Mev}$ may be somewhat higher, and that at 300 Mev somewhat lower, than indicated in Table VIII; the $\left[\mathrm{He}^{37}\right.$ results affect the $\left[\mathrm{He}^{3}\right]$ and $\left[\mathrm{He}^{4} /\right.$ cross sections and the ratio of the inelastic to the total cross section. It is perhaps worth noting that, with the assumption of an $A^{2 / 3}$ dependence, a straight-line extrapolation of Ball's measurements 29 of the inelastic cross section for $300-\mathrm{Mev}$ neutrons on $\mathrm{Pb}, \mathrm{Cu}, \mathrm{Al}$, and $\mathrm{C}$ targets gives $\sigma_{\text {inel }}=70 \pm 10 \mathrm{milli}-$ barns for helium. ${ }^{5}$ From the straight-line fits by Millburn et al. 30 
to the experimental inelastic cross sections at $300 \mathrm{Mev}$ (mainly the data of Ball) one can calculate the inelastic cross section for helium as 79 millibarns. Nedzel 21 has fitted the 270 . to $280-\mathrm{Mev}$ total cross sections to the transparent optical model of Fernbach, Serber, and Taylor; 31 if the constants so determined a re applied to helium the calculated total cross section is 108 millibarns and the inelastic cross section is 82 millibarns. This experiment, which normalized the data to a total cross section of 100 millibarns, finds an inelastic cross section of $70.2 \pm 5.2$ millibarns.

Moulthrop, by invoking the principle of charge symmetry, has formulated a comparison among the inelastic cross sections for the several processes observed by Tannenwald (meson production not in volved) and the relative cross sections observed in his own experiment for the corresponding processes involving negative-pion production; ${ }^{5}$ the comparison was made for all processes except the pion-producing reaction $\mathrm{He}^{4}\left(\mathrm{n}, \mathrm{p} \pi^{-}\right) \mathrm{He}^{4}$, which is the analogue of the true elastic process $\mathrm{He}^{4}(n, n) \mathrm{He}^{4}$. The following table exlends that originally prepared by Moulthrop, in comparing his own and Tannenwald's experiment, to include this experiment. 
Table IX

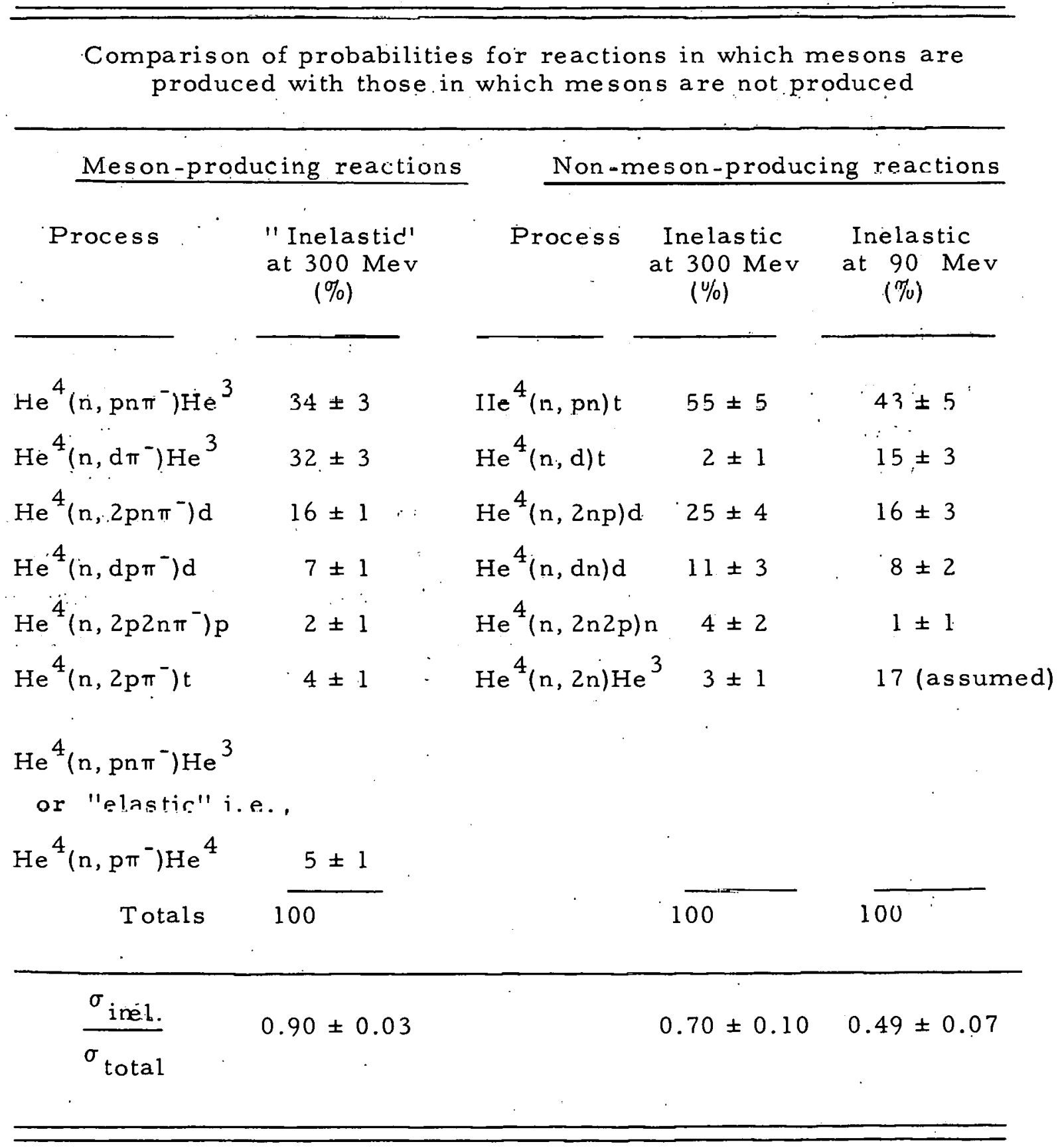


In comparing his own and Tannenwald's experiments, the second and fourth columns above, Moulthrop called attention to the striking agreement in "inelastic" cross sections and noted that the "inelastic" cross sections, other than $\mathrm{He}^{4}\left(\mathrm{n}, \mathrm{pn} \pi^{-}\right) \mathrm{He}^{3}$ and $\mathrm{He}^{4}\left(\mathrm{n}, \mathrm{d} \pi^{-}\right) \mathrm{He}^{3}$, could be interpreted by the same sort of arguments as needed to understand the $90-\mathrm{Mev}$ cross sections, and were not appreciably influenced by the production of a meson. Examination of the second and third columns of Table IX reveals that the analogy is not so striking at $300 \mathrm{Mev}$, but that it is cerlainly still qualitatively true.

Moulthrop also noted a real correlation between pion production and fast-deuteron formation ("fast" being applied to a particle of energy greater than $50 \mathrm{Mev}$. He observed the ratio "Fast Deuterons/ (Fast Deuterons + Fast Protons)" to have the value of $0.38 \pm 0.06$ for negative-pion production in helium at $300 \mathrm{Mev}$, and cited the results of Ford ${ }^{32}$ and Knapp ${ }^{33}$ as $0.31 \pm 0.06$ and 0.5 (estimated) in similar pion-production experiments at $300 \mathrm{Mev}$ for oxygen and deuterium re spectively. The corresponding ratio for this experiment, without pion production, is 0.16 , a value which tends to confirm the definite correlation noted by Moulthrop.

\section{Energy and Angular Distributions}

The $\mathrm{He}^{4}(\mathrm{n}, \mathrm{pn}) \mathrm{t}$ Reaction. Heidmann's predictions for this reaction at $90 \mathrm{Mev}$ and $200 \mathrm{Mev}$ are that the tritons are of low energy and emitted almost isotropically in the laboratory system. ${ }^{6}, 7$ At $90 \mathrm{Mev}$ Tannenwald found that the angular distribution was not isotropic but concentrated in the forward direction; he found the triton energy distribution in excellent agreement with the prediction. ${ }^{1}$ At $200 \mathrm{Mev}$ Swartz observed that the distribution did not seem compatible with the predicted isotropic distribution. ${ }^{3}$ The laboratory-system angular distribution of tritons in this experiment is shown in Fig. 1. It is very similar to that found by Swartz for all prongs; he was unable to deduce a distribution that could be definitely called that of tritons from $\langle\mathrm{PT}\rangle$ alone. Figure 1 suggests a concentration in the forward direction, but not to the marked extent 
observed by Tannenwald at $90 \mathrm{Mev}$. Figure 2 shows the energy distribution of the tritons in this experiment; agreement with prediction for the lower energies is excellent

The proton angular distribution from /PT/ events is shown in Fig. 3; it is very similar to that observed by Tannenwald at $90 \mathrm{Mev}$. Figure 4 shows the energy distribution of protons. In contrast to the observations of Tannenwald, at $90 \mathrm{Mev}$, that the number of protons per 20 -Mev energy interval was maximum in the 0 - to 20 -Mev interval, and decreased steadily with energy, the distribution here is qualitatively suggestive of that which would be found for recoil protons in free $n-p$ elastic scattering. This is illuslraled by the superimposed surve, which is the nonrelativistic $N(E)$ vs $E$ for recoil protons from $340-\mathrm{Mev}$ neutrons, normalized to the total number of events comprising the histog ram.

The $\mathrm{He}^{4}(\mathrm{n}, 2 \mathrm{np}) \mathrm{d}$ Reaction. Figures 5 and 6 show the laboratorysystem angular distribution and energy distribution, respectively, for the deuterons emitted in $[P D]$ events; the deuterons tend to peak in the forward direction and to be of low energy. Figures 7 and 8 show the corresponding distributions for the protons of $\angle P D /$ events. The protons tend to peak in the forward direction; in view of the poor statistics it is doubtful that the pronounced peak in the energy distribution for 120 to $160 \mathrm{Mev}$ j.s real.

Other Inelastic Reactions. The $\langle\mathrm{DT}\rangle,\langle\mathrm{DD}\rangle,\langle\mathrm{PP}\rangle$, and $\left[\mathrm{He}^{3}\right\rangle$ reactions are of infrequent occurrence and there are not sufficient data for an attempt at determination of energy and angular distributions.

Elastically Scattered Neutrons. Heidmann has predicted the angular distribution in the center-of-mass system for neutrons elastically scattered by helium at $90 \mathrm{Mev}$ and $200 \mathrm{Mev}$. The theoretical predictions are gaussian and centered on the forward direction, and are given by 


$$
\begin{aligned}
\frac{\mathrm{d} \sigma}{\mathrm{d} \Omega} & =450 \mathrm{e}^{-7.86 \dot{\theta}^{7}} \text { millibarns } \quad(90 \mathrm{Mev}) \\
& =450 \mathrm{e}^{-17.5 \theta^{2}} \text { millibarns. }
\end{aligned}
$$

with angular half widths of about $17^{\circ}$ and $11^{\circ}$ respectively. These predictions show a rapid trend toward increasingly sharp peaking in the forward direction with increasing energy; with regard to the $200-\mathrm{Mev}$ prediction, however, Heidmann states that, although the equation shows that only about 1 in $10^{7}$ are scattered to the rear, this particular result should be considered as valueless because of the neglect of the Fourier components curresponding. to large changes of momentum and the use of Gaussian functions to permit analytic integrations.

. Tannenwald found the 90-Mev experimental data not incompatible with a Gaussian distribution, and obtained a good fit by using an exponent of $-5 \theta^{2}$. The angular half width corresponding to this equation is about $21^{\circ}$.

The angular distribution $\mathrm{d} \sigma / \mathrm{d} \Omega$ (in the center-of-mass system) of elastically scattered neutrons, from incident neutrons of energy equal to or greater than $160 \mathrm{Mev}$, found in this experiment is shown in Fig. 9. These data are also not incompatible with a Gaussian, and a good fit is obtained with the superimposed curve whose exponent is $-5.4 \theta^{2}$; the angular half width is again about $21^{\circ}$, as found by Tannenwald at $90 \mathrm{Mev}$. These data are also shown in Fig. 10 as $\mathrm{d} \sigma / \mathrm{d} \theta=(\mathrm{d} \sigma / \mathrm{d} \Omega) \sin \theta$ and the corresponding curve for the empirical Gaussian fit has been superimposed. As described in Chapter V, Results and Discussion, the latter curve was used to correct the elastic data for tracks missed because they were too short to be measured.

It appears from the $90 \mathrm{-Mev}$ data of Tannenwald and the data of this experiment that the sharpness of the forward peak is substantially less than predicted by. Heidmann and that the change between $90 \mathrm{Mev}$ and $300 \mathrm{Mev}$ is much less than would be expected from Heidmann's calculations. 
Energy of Incident Neutrons. Figure 11 shows the energy distribution of the incident neutrons as derived from the elastic-scattering data; Fig. 12 shows this distribution as derived from the $[\mathrm{PT}, /[\mathrm{DD} /$ and $[\mathrm{DT}]$ data. Since $[\mathrm{PD}],\langle\mathrm{PP}\rangle$, and $\left[\mathrm{He}^{3 /}\right.$ permit calculation of a minimum neutron energy only, they have not been employed in the se distributions. The energy spectrum of the incident-neutron beam given in Fig. 13 for a 1/2-inch LiD target is due to Ball, ${ }^{29}$ and has been refined by De Pangher. ${ }^{34}$ The smooth curve superimposed on F'ig.. 11 is obtained from the results of De Pangher normalized to the total number of events comprising the histogram. It is seen that the results of this experiment indicale a significantly greater number of neutrons in the 160 - to $200-\mathrm{Mev}$ interval than should be expected from the work of Ball and De Pangher. Although the similar curve has not been superimposed on Fig. 12 a greater number of 160 - to $200-\mathrm{Mev}$ neutrons is found here also. The statistics are poor in both cases.

\section{Oxygen Stars}

Because of the presence of water vapor in the cloud chamber a few oxygenstars were observed. Oxygen stars of two or more prongs originating in the acceptable region of the chamber, as described in: Chapter III, Methnd if Analysis of Events, were recorded without the imposition of any restriction on dip angle. Their distribution, according to the number of prongs in the star, is given in the following table; the percentage distribution is also included together with that observed by Fuller in a study of the disintegration of oxygen by $300-\mathrm{Mev}$ neutrons. 35 
Table X

Distribution of oxygen stars originating in cloud chamber under neutron bombardment

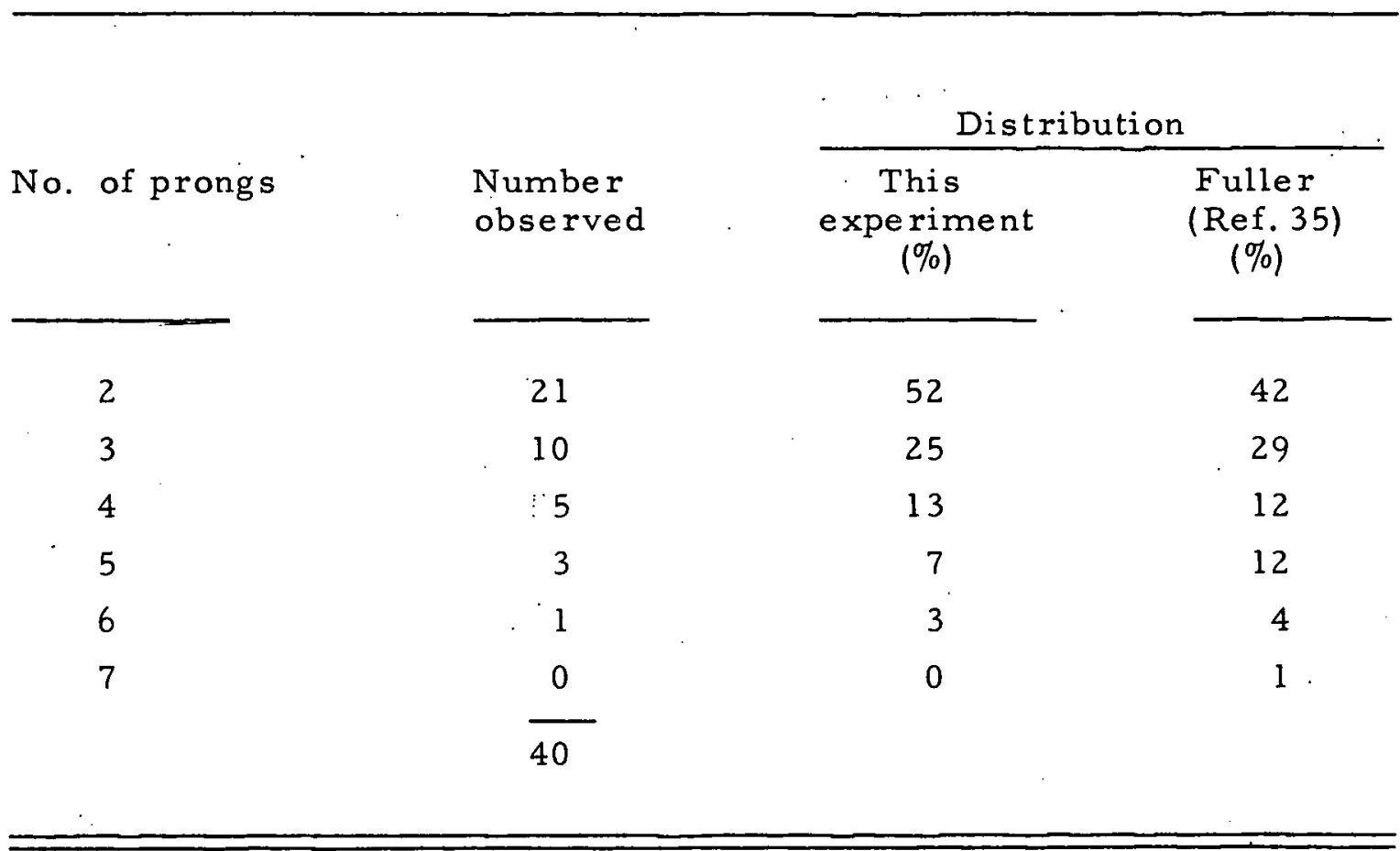

The number observed can be compared with the number expected on the basis of the number of inelastic helium events observed, the inelastic cross sections of helium and oxygen, and the ratio of helium. to oxygen nuclei in the cloud chamber. Since no dip-angle limitation was imposed on the oxygen stars, and no calculations were made of the energy of the incident neutrons involved, the number of inelastic helium events observed at all angles and energies and without weighting should be used; this number is 473 . The inelastic cross section for helium is 70 millibarns from this experiment; interpolation in the data of Ball gives 255 millibarns for the inelastic cross section of oxygen. 29 The ratio of helium to oxygen nuclei in the chamber was 51.8. The calculated expected number of inelastic oxygen stars is 33 compared with the 40 that were actually obse rved. 


\section{Azimuthal Symmetry Check}

It has been assumed that all processes in this experiment occurred with azimuthal symmetry, and this assumption has been the basis for the geometric correction factor applied in weighting the observed events. It is desirable to verify this assumption by an examination of some of the azimuthal distributions involved. The following table shows the number of $\left[\mathrm{He}^{4} /\right.$ recoils, and tritons from $\left./ \mathrm{PT}\right]$, actually observed in four azimuthal angular intervals.

Table XI

Azimuthal distribution of $\left[\mathrm{He}^{4}\right]$ recoils and tritons from $[\mathrm{PT} /$

Azimuthal angular interval

\begin{tabular}{l|rrrrr}
$\mathrm{He}^{4}$ recoils & $19 \pm 6$ & $30 \pm 7$ & $31 \pm 7$ & $23 \pm 6$ \\
Tritons from & $32 \pm 7$ & $37 \pm 8$ & $32 \pm 7$ & $24 \pm 6$
\end{tabular}

The uncertainties are standard deviations based on the number of evento actually observed. 


\section{CONCLUSIONS}

In the bombardment of helium by $300-\mathrm{Mev}$ neutrons the dominant reaction is inelastic scattering, which accounts for $70 \%$ of the total cross section. Of the six possible inelastic reactions (exclusive of meson-producting. reactions) the most frequent is $[\mathrm{PT}]$, in which the incident neutron strips a proton from the helium nucleus, leaving a low-energy triton; [PT] accounts for $55 \%$ of the inelastic cross section. The phenomenological analogue of this process, $\angle \mathrm{He}^{37}$, in which the incident neutron strips a neutron from the helium nucleus and leaves a low -energy $\mathrm{He}^{3}$ nucleus, is negligible at this energy and accounts for about $3 \%$ of the inelastic cross section. The $\angle \mathrm{DT} 7$ process is also negligible at this energy, accounting for about $2 \%$ of the inelastic cross section. The/ $/ \mathrm{PD} /$ process contributes $25 \%$, and the $[\mathrm{DD} /$ process $11 \%$, of the inelastic cross section. The complete disintegration of helium, $\angle P P^{\prime}$, is rare and contributes about $4 \%$ of the inelastic cross section. With the exception of $\left[\mathrm{DT} /\right.$ and $\left(\mathrm{He}^{3}\right)$ these results are similar to those found in a similar experiment at $90 \mathrm{Mev} .^{1}$ At the lower energy about half the $[\mathrm{DT}]$ cross section was contributed as a special case of $\left[\mathrm{PT}_{1}^{7}\right.$ through the pickup process in which the proton and outgoing neutron form a high-energy forward deuteron; the pickup portion of $/ \mathrm{DT} 7$ would be expected to be neglible: at $300 \mathrm{Mev}$. A direct comparison with theory is not possible, but qualitative comparisons may be made with pre dictions made for $90 \mathrm{Mev}$ and $200 \mathrm{Mev}$. 6,7 Such comparisons reveal agreement in the cross section for $\langle\mathrm{PT}\rangle$, but not in the angular distri.bution of the associated tritons, and indicates that the theory probably greatly underestimates the frequency of $/ \mathrm{PD}]$ and $/ \mathrm{DD}]$; similar conclusions obtained for the $90 \mathrm{-Mev}$ experiment.

Elastic scattering exhibits the expected forward peak of scattered neutrons in the center-of-mass system. The differential cross section for elastic scattering is not incompatible with a gaussian distribution, of angular half width about $21^{\circ}$, and is quite similar to that found in the 90-Mev experiment. The sharpness of the forward peak is considerably less than would be expected from qualitative extrapolation of the available theoretical predictions. 
A small cross section for negative-pion production in helium, and some evidence supporting a possible correlation between pion production and fast deuteron formation, ${ }^{5}$ have been observed. 


\section{ACKNOWLEDGMENTS}

The author wishes to acknowledge his indebtedness to Professor Wilson M. Powell for suggesting the experiment and for making available the extensive facilities of the University of California Radiation Laboratory cloud chamber group; and particularly for his counsel, continuing interest, and active participation throughout the investigation.

Many members and former members of the cloud chamber group contributed in one way or another to the completion of this experiment, and their cooperation is acknowledged with pleasure. Special thanks are due to Dr. Franklin C. Ford, Dr. Chung Y ing Chih, and Messrs. John B. Elliott, Larry O. Oswald, and Milton M. Hill, who furnished valuable technical assistance. Mrs. Dorothy J. Gardner developed the stereoscopic negatives.

Special thanks are also due to Mr. S. C. Hight, Direction of Research, Sandia Corporation, for making available the computer facilities of the Sandia Corporation, and to Miss M.K. Weston and Mrs. E.G. Young, who programmed the computations and ran them on the CRC $102-\mathrm{A}$ computer.

This work was performed under the auspices of the Atomic Ene rgy Commission. 


\section{A PPEN.DIX \\ I. DEFINITIONS}

Dip angle a

Beam angle $\beta$

Scatter angle $\theta$

Azimuthal angle $\phi$

Slant radius $\rho_{s}$

Radius $\rho$

Slant plane

Transverse momentum

Momentum in the beam direction
The angle between the initial direction of the track and its projection on the horizontal plane containing the neutron beam.

The angle between the projection of the initial track direction on the horizontal plane and the direction of the neutron beam.

The angle between the initial track direction and the neutron beam:

The angle between the projection of the initial track direction on a plane perpendicular to the neutron beam, and the horizontal plane.

The radius of curvature of the track as measured in the slant plane.

$\rho=\rho_{s} \cos a:$ is the radius of curvature that a particle of slant radius $\rho_{s}$ would have if it were nuving with the same momentum in a plane perpendicular to the magnetic field.

The plane containing the initial track direction and the horizontal line perpendicular to the initial track direction. It is approximately the plane of the track except that, in general, the path of a charged particle in a magnetic field describes a helix. The slant plane is at dip angle a to the horizontal: plane.

$H \rho_{t}=H \rho \sin \theta$

$\mathrm{H} \rho_{\mathrm{z}}=\mathrm{H} \rho \cos \theta$ 


\section{DERIVATION OF FORMULAS}

\section{General Formula for All Reactions Except [DT]}

Consider the collision of a fast particle (neutron) of kinetic energy $T_{n}$, total energy $E_{n}$, and rest mass $m$, with a second particle at rest (helium nucleus) of total energy $E_{M}$ and rest mass. $M$. After the collision we observe charged particles Nos. 1 and 2 of kinetic energy $T_{1}$ and $T_{2}$, total energy $E_{1}$ and $E_{2}$, and rest mass $m_{1}$ and $\mathrm{m}_{2}$. Present, but not visible, is the path of an uncharged particle of kinetic energy $T_{n^{\prime}}$, total energy $E_{n} ;$ and rest mass $m^{\prime}$.

Introduce a set of rectangular coordinates with the positive direction of the $z$ axis coinciding with the momentum vector of the incidentparticle; let subscript $z$ denote $z$ components of momentum and subscript $t$ denote transverse components of momentum. Conservation of total energy and momentum requires

$$
\begin{aligned}
E_{n}+E_{M} & =E_{1}+E_{2}+E_{n^{\prime}}, \\
P_{n} & =p_{1 z}+p_{2 z}+p_{n^{\prime} z} \\
0 & =p_{1 t}+p_{2 t}+p_{n^{\prime} t},
\end{aligned}
$$

where $p^{2}=p_{z}^{2}+p_{t}^{2}$ for each particle.

These equations are just sufficient to determine the three unknowns of the problem, namely, $p_{n^{\prime} z}$ and $p_{n^{\prime} t}$ (whence $p_{n^{\prime}}$ and $E_{n^{\prime}}$ ) and $P_{n}$ (whence $E_{n}$ ). Denoting $E_{1}+E_{2}-E_{M}$ by $\Sigma$ in the first of the above equations; and $\mathrm{p}_{1 \mathrm{z}}+\mathrm{p}_{2 \mathrm{z}}$ by $\mathrm{P}$ in the second; and introducing the relativistic relation between total energy and momentum, we can write

$$
\begin{aligned}
& E_{n}=\Sigma+E_{n^{\prime}}, \\
& E_{n}^{2}=c^{2} p_{n}^{2}+\left(m c^{2}\right)^{2}, \\
& E_{n^{\prime}}^{2}=c^{2} p_{n^{\prime}}^{2}+\left(m^{\prime} c^{2}\right)^{2} .
\end{aligned}
$$


Squaring Eq. (1) and substituting the resulting expression for $\mathrm{E}_{\mathrm{n}}^{2}$ into Eq. (2) gives

$$
\Sigma^{2}+2 E_{n^{\prime}} \quad \Sigma+E_{n^{\prime}}^{2}=c^{2}\left(P+p_{n^{\prime} z^{\prime}}\right)^{2}+\left(m c^{2}\right)^{2}
$$

Substituting $E_{n^{\prime}}$ and $E_{n^{\prime}}^{2}$ from $E q$. (3) into (4) gives, after considerable simplification, a solution of the quadratic equation in $\mathrm{P}_{\mathrm{n}^{\prime} \mathrm{z}}$ as follows:

$$
\begin{aligned}
& p_{n^{\prime} z}=\frac{e}{c} \frac{(z H \rho) z\left\{9(z H \rho)_{z}^{2}-9(z H \rho)_{t}^{2}+10^{8}\left[\left(m c^{2}\right)^{2}-\left(m^{\prime} c^{2}\right)^{2}-\Sigma^{2}\right]\right\}}{2 \cdot\left[10^{8} \Sigma^{2}-9(z H \rho)_{z}^{2}\right]} \\
& \pm \frac{\mathrm{e}}{\mathrm{c}} \frac{10^{4}}{3} \mathrm{~s} \frac{\left[\begin{array}{l}
\left.\left\{9(\mathrm{zH} \rho)_{\mathrm{z}}^{2}-9(\mathrm{zH} \rho)_{\mathrm{t}}^{2}+10^{8}\left[\left(\mathrm{mc}^{2}\right)^{2}-\left(\mathrm{m}^{\prime} \mathrm{c}^{2}\right)^{2}-\Sigma^{2}\right]\right\}\right] \\
-4\left\{10^{8} \Sigma^{2}-9(\mathrm{zH} \rho)_{z}^{2}\right\}\left\{9(\mathrm{zH} \rho)_{\mathrm{t}}^{2}+10^{8}\left(\mathrm{~m}^{\prime} \mathrm{c}^{2}\right)^{2}\right\}
\end{array}\right]}{2\left[10^{8} \Sigma^{2}-9(\mathrm{zH} \rho)_{\mathrm{z}}^{2}\right]}
\end{aligned}
$$

where all energies are in Mev, $\mathrm{P}_{\mathrm{n}^{\prime} \mathrm{z}}$ is in Mev/c, the notation $(z \mathrm{H} \rho)_{z}$ is equivalent to $\left(z_{1} \mathrm{H}_{1} \rho_{1}\right)_{z}+\left(z_{2} \mathrm{H}_{2} \rho_{2}\right)_{z}$, and similarly for $\left(z \mathrm{H}_{\rho}\right)_{t}$ where $z_{1}$ and $z_{2}$ are the particle charge numbers.

This result can be written in the symbolic form,

$$
\frac{c}{\mathrm{e}} \mathrm{p}_{\mathrm{n}^{\prime} \mathrm{z}}=\frac{\mathrm{A}(\mathrm{H} \rho) \mathrm{z}\left[\mathrm{J} \pm \frac{10^{4}}{3} \Sigma[]^{2}-4 \Delta \sigma\right.}{2 \Delta} \text {, }
$$

where $A$ is either 1 or 2 and []$, \Delta$, and $\sigma$ are all functions of $\Sigma$, $(\mathrm{H} \rho)_{z}$, and $(\mathrm{H} \rho)_{t}$. A program involving only these three quantities as inputs can be prepared for solution by an automatic computer.

Considerable numerical simplification is possible if it is kept in mind that: for [PT] and $[\mathrm{DD} /$ only one neutron is ejected so that one has $\mathrm{m}^{\prime}=\mathrm{m}$; for $[\mathrm{PD}]$ two neutrons are ejected, but can be lumped for a minimum-energy solution, so that one has $\mathrm{m}^{\prime}=2 \mathrm{~m}$; for $[\mathrm{PP}]$ three 
neutrons are ejected, but can be lumped for a minimum solution, so that one has $\mathrm{m}^{\prime}=3 \mathrm{~m}$; for $\left[\mathrm{He}^{3} /\right.$ only one charged particle is involved, so that one has $m_{2}=0$, and two neutrons are ejected so that one has $\mathrm{m}^{\prime}=2 \mathrm{~m}$ for a minimum solution; for $\angle \mathrm{He} / \mathrm{4}$ only one charged particle is involved, so that one has $\mathrm{m}_{2}=0$, and only one neutron is ejected so that one has $m^{\prime}=m$; and finally, for all cases,

$$
\Sigma=E_{1}+E_{2}-E_{M}=T_{1}+T_{2}+m_{1} c^{2}+m_{2} c^{2}-M c^{2} .
$$

The computer obtains $\frac{c}{e} p_{n^{\prime} z}$ in units of gauss $-\mathrm{cm} \times 10^{-5}$, solves for $T_{n}$, and $T_{n}$ in Mev, and prints out the results. Sample calculation sheets, showing the data read from the cloud chamber photographs, the derived computer inputs, and the computer results appea $r$ at the end of this Appendix.

\section{Formulas for the $/ \mathrm{DT} 7$ Reaction}

This is the two-body problem and $T_{n}$ can be calculated in two ways:

$$
\begin{aligned}
& T_{n}=T_{1}+T_{2}+B E \\
& T_{n}=m_{0} c^{2}\left[\sqrt{1+\frac{c^{2} p^{2}}{\left(m_{0} c^{2}\right)^{2}}}-1\right] .
\end{aligned}
$$

The second solution is obtained with sufficient accuracy by use of the alignment chart for protons (Fig. 18), taking $p$ in gauss-cm as the sum of the observed $(\mathrm{H} \rho)_{z}$ values of the deuteron and triton.

\section{Formulas for $\left[\mathrm{He}^{4} /\right.$ Neutron-Scattering Angle}

It can be easily shown that the scattering angle of the elastically scattered neutron in the center-of-mass system is related to the recoil angle of the helium nucleus in the laboratory system by

$$
\tan \theta=\sqrt{1-\beta^{2}} \cot \frac{\theta^{\prime}}{2},
$$


where $\theta$ is the recoil angle of the helium nucleus in the laboratory system, $\theta^{\prime}$ is the corresponding scatter angle of the neutron in the center-of-mass system, and $\beta=v / c$ for the velocity of the center-of mass system relative to the laboratory system. For $n-\mathrm{He}^{4}$ elastic scattering one has

$$
1-\beta^{2}=\frac{8\left(\frac{T_{n}}{m_{0} c^{c}}+1\right)+17}{\left(\frac{T_{n}}{m_{0} c^{2}}+5\right)^{2}}
$$

Equation (A I) is easily solved by a simple circular nomogram with a family of indices, determined from Eq. (A2), for $T_{n}=200,250,300$, and $350 \mathrm{Mev}$.

\section{Binding Ene rgies}

$\begin{array}{cc}\text { Process } & \begin{array}{c}\text { Binding Energy } \\ \text { (Mev) }\end{array} \\ \langle\mathrm{PT}\rangle & 19.8 \\ \angle \mathrm{DT}\rangle & 17.6 \\ \angle \mathrm{PD} 7 & 26.0 \\ \angle \mathrm{DD}\rangle & 23.8 \\ \angle \mathrm{PP}\rangle & 28.1 \\ \left.\angle \mathrm{He}^{3}\right\rangle & 20.5\end{array}$




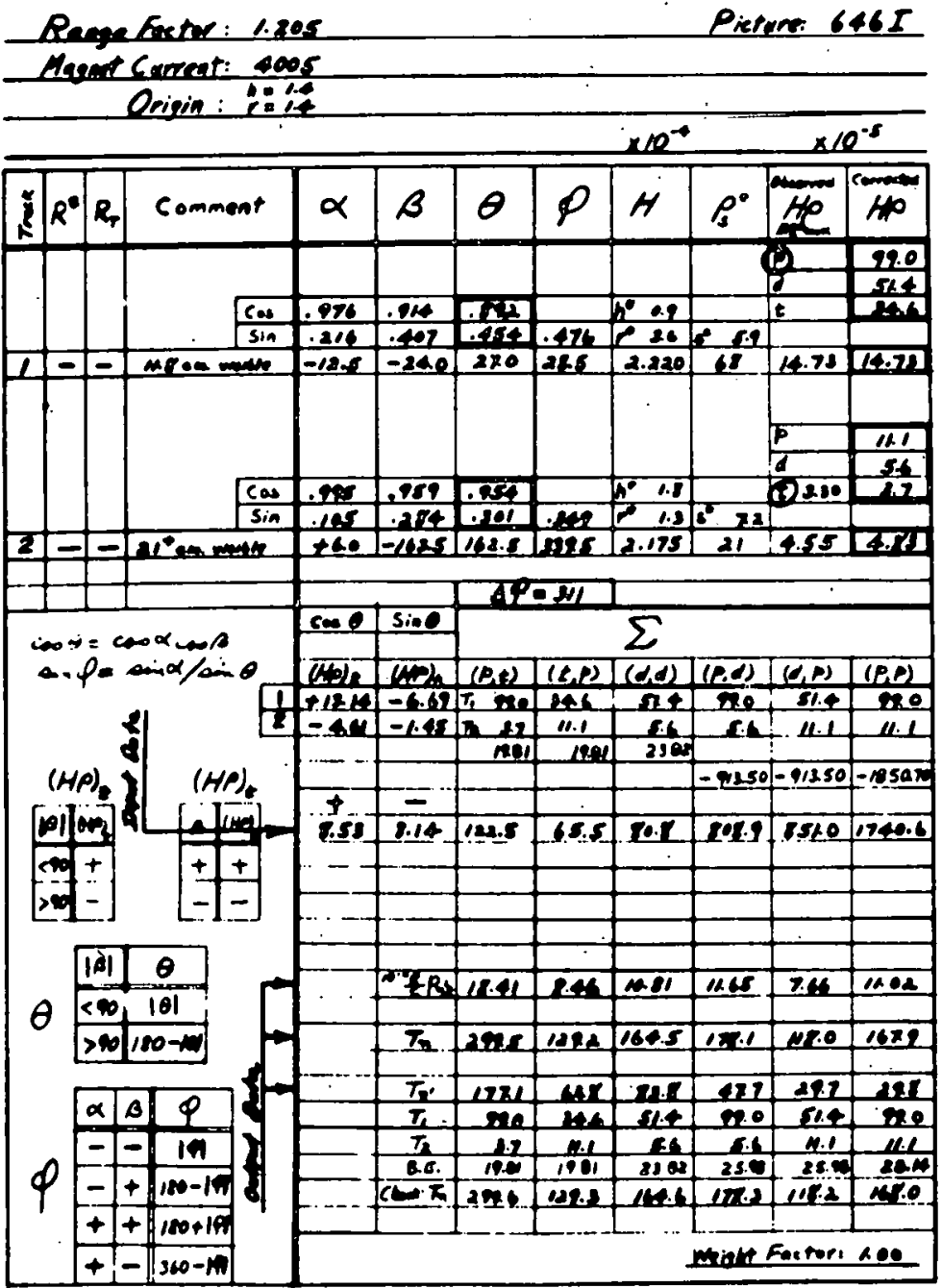

$M U-14473$ 


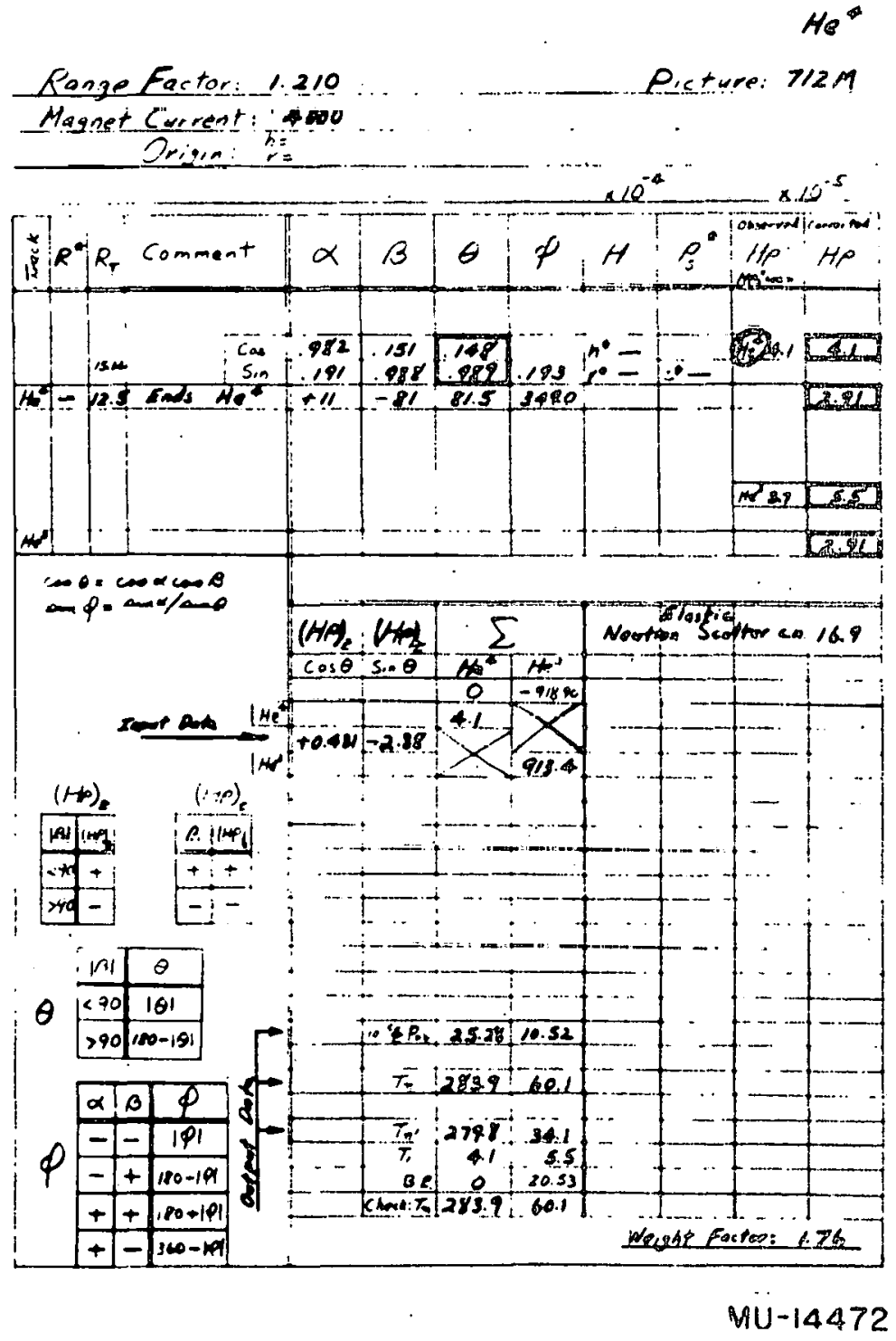




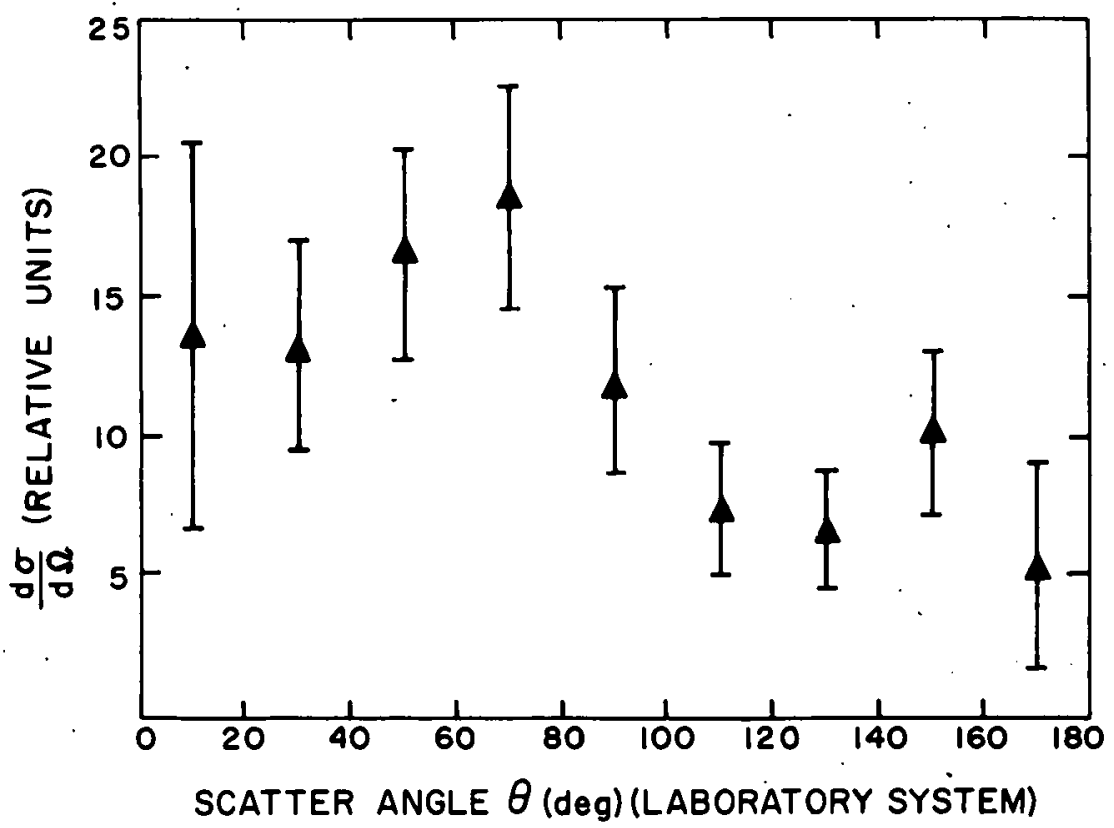

$M U-14358$

Fig. 1. Angular distribution of tritons from $\langle P T\rangle$ events. 


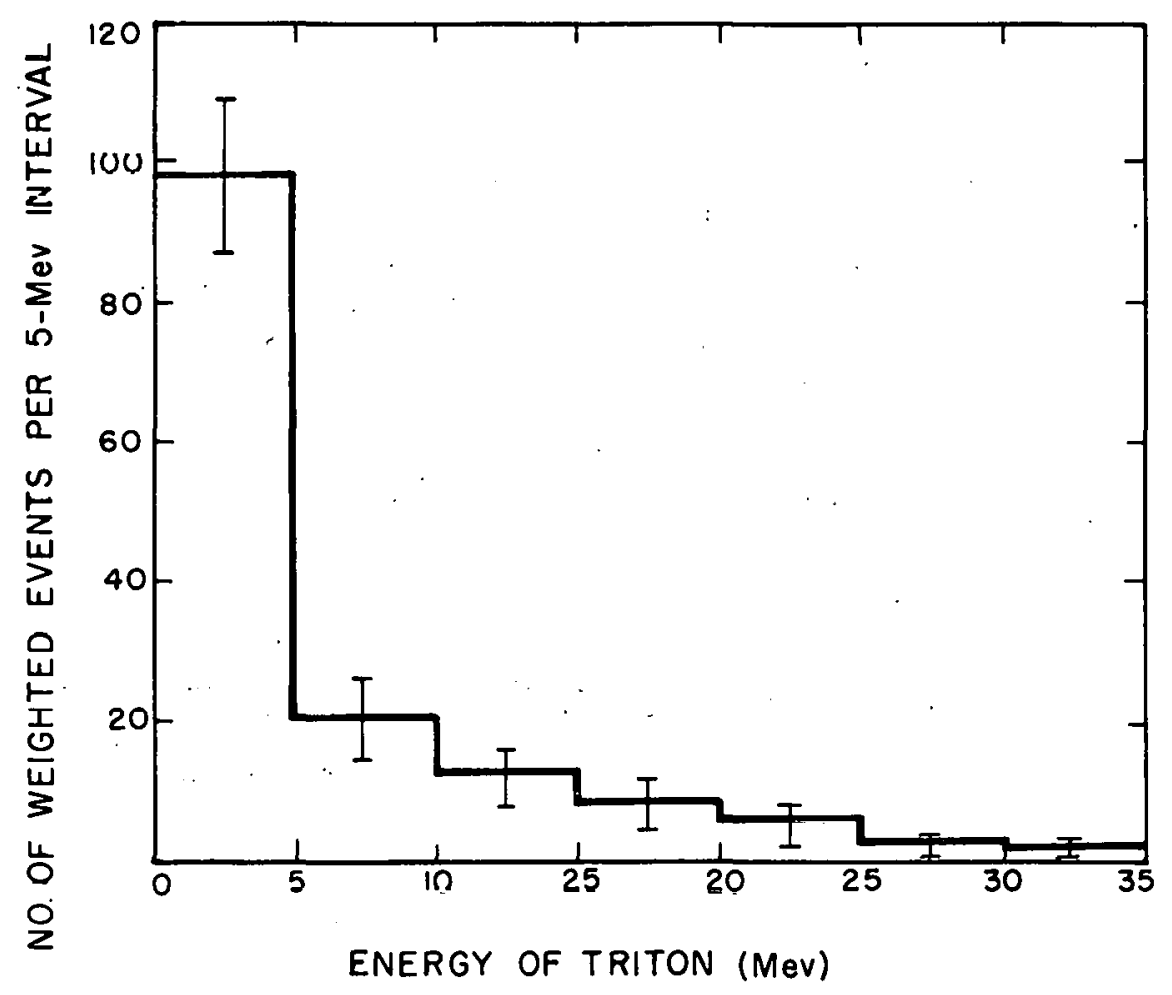

$M U-14359$

Fig. 2. Energy distribution of tritons from $\lfloor P T 7$ events. 


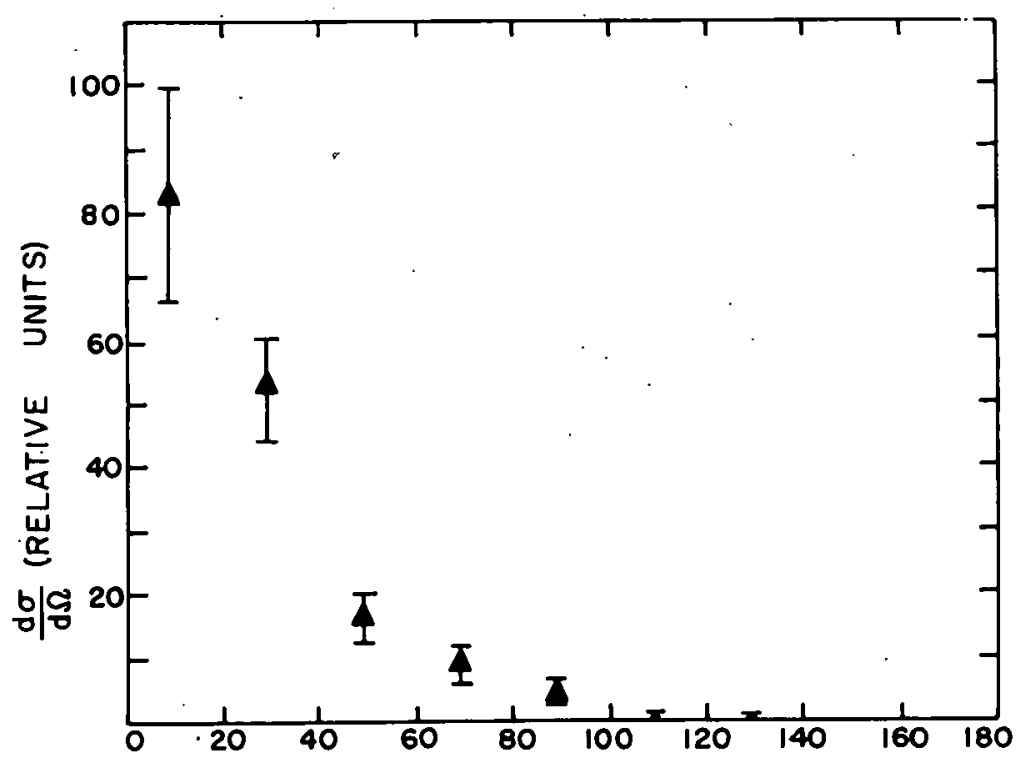

SCATTER ANGLE $\theta$ (deg) (LABORATORY SYSTEM)

MU-14360

Fig. 3. Angular distribution of protons from $\angle P T\rangle$ events. 


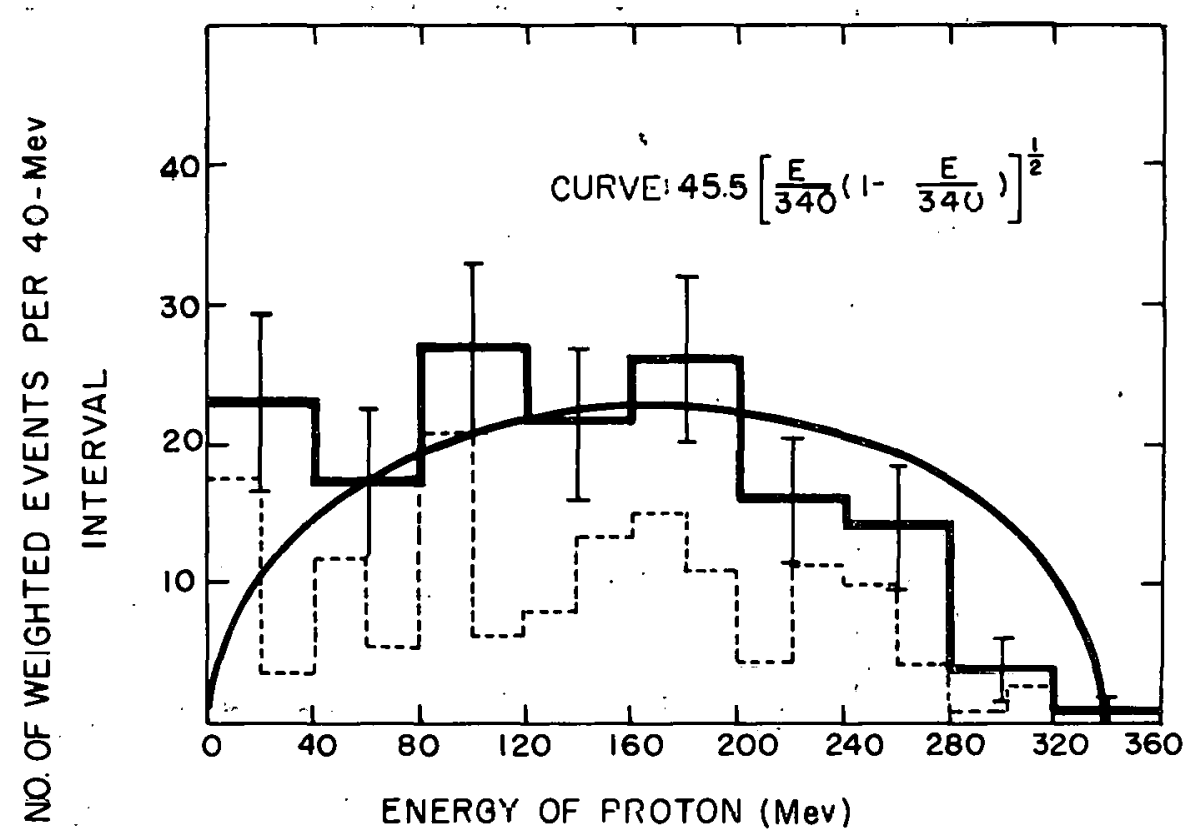

$M U-14361$

Fig. 4. Energy distribution of protons from $\lfloor\mathrm{PT}\rceil$ events。 


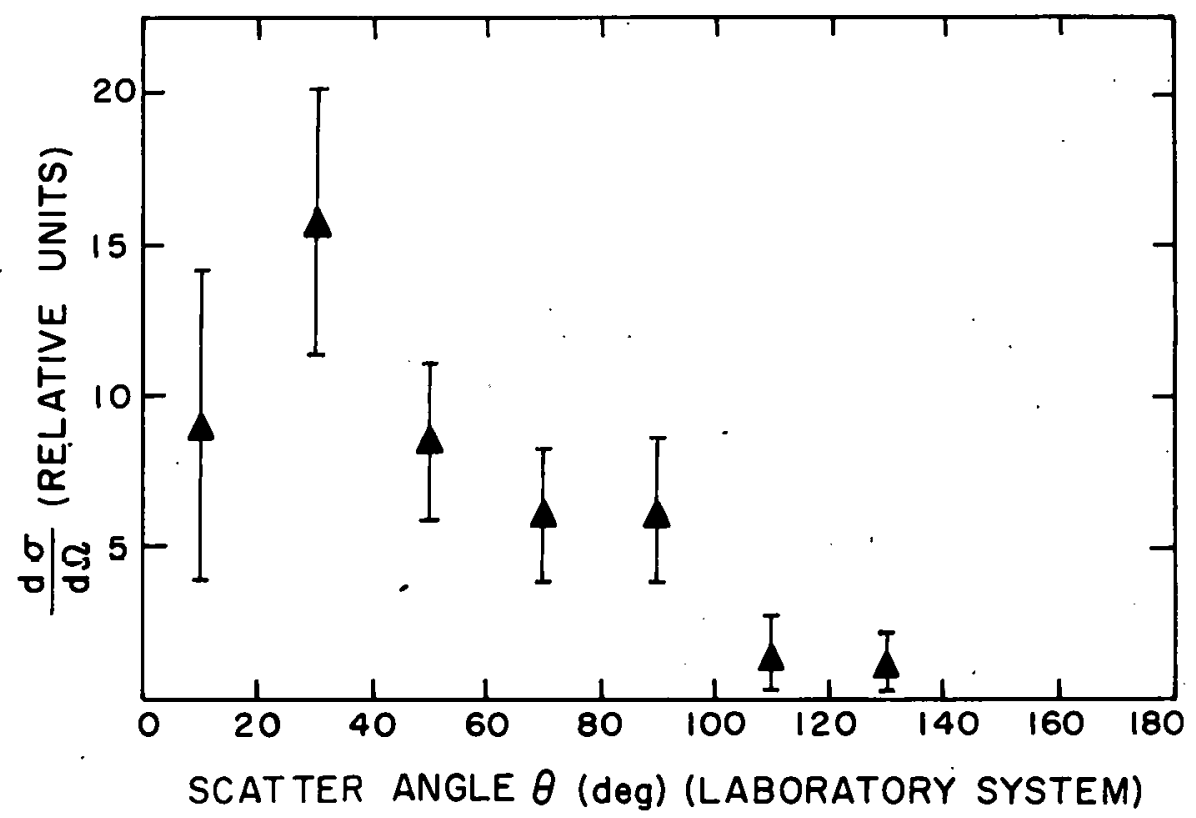

$M U-14362$

1 Fig. 5. Angular distribution of deuterons from $[\mathrm{PD}]$ events. 


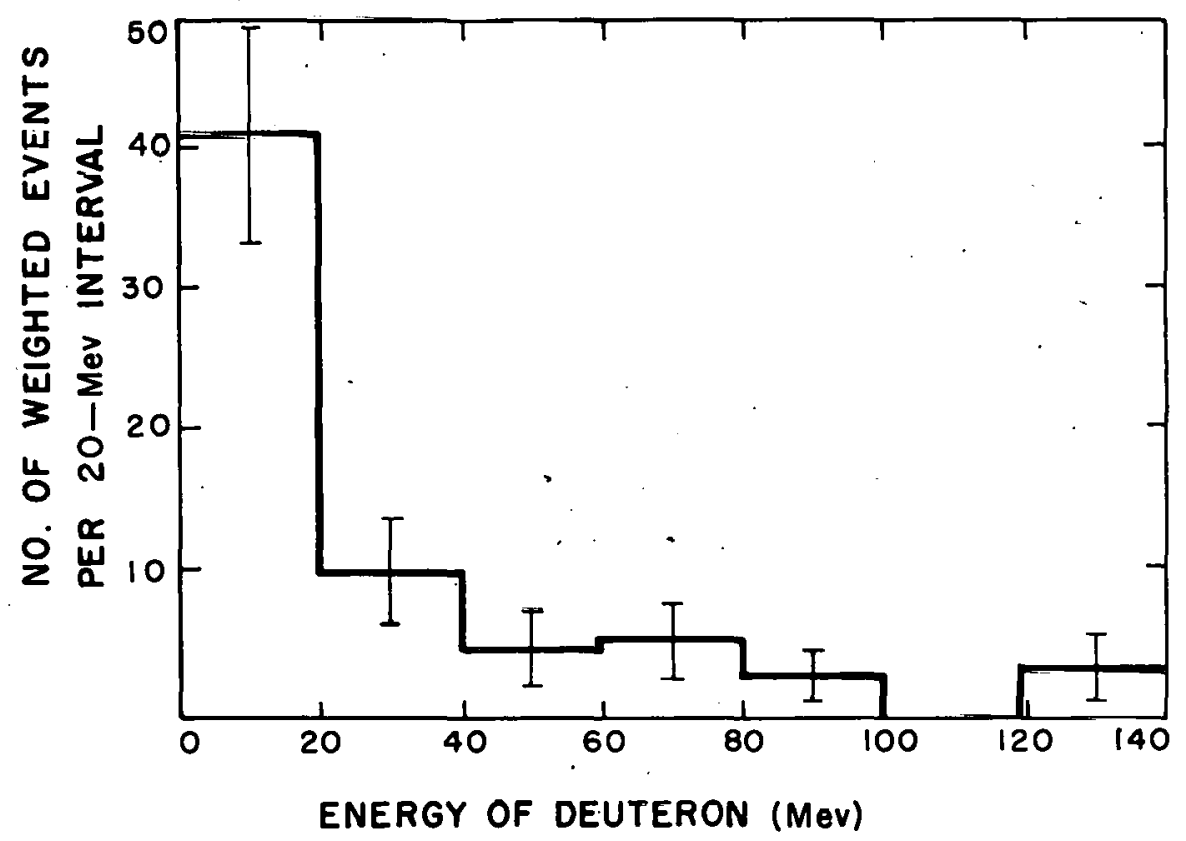

MU-14363

Fig. 6. Energy distribution of deuterons from $[P D]$. events. 


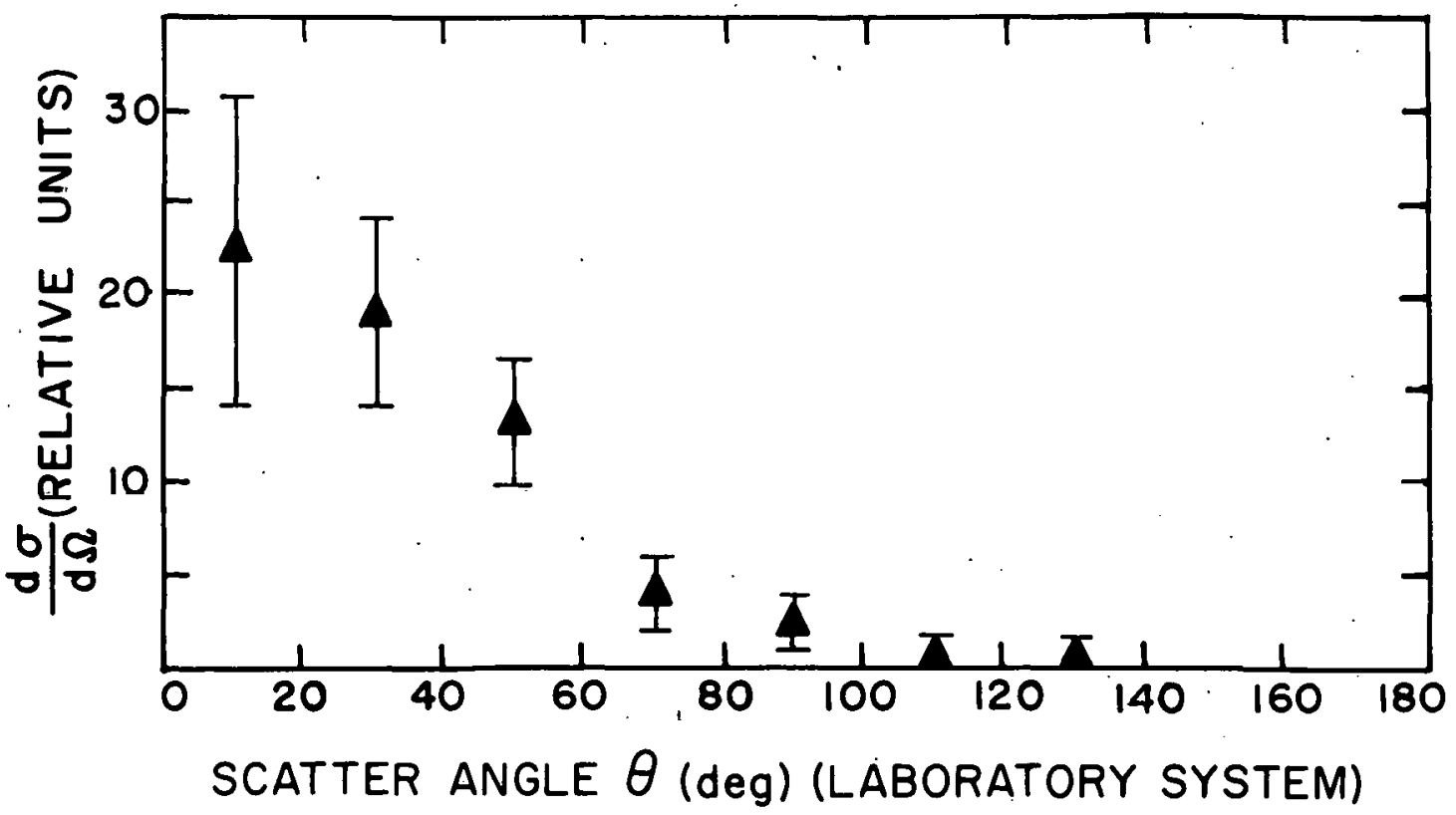

$M U-1436.4$

Fig. 7. Angular distribution of protons from $[P D]$ events. 


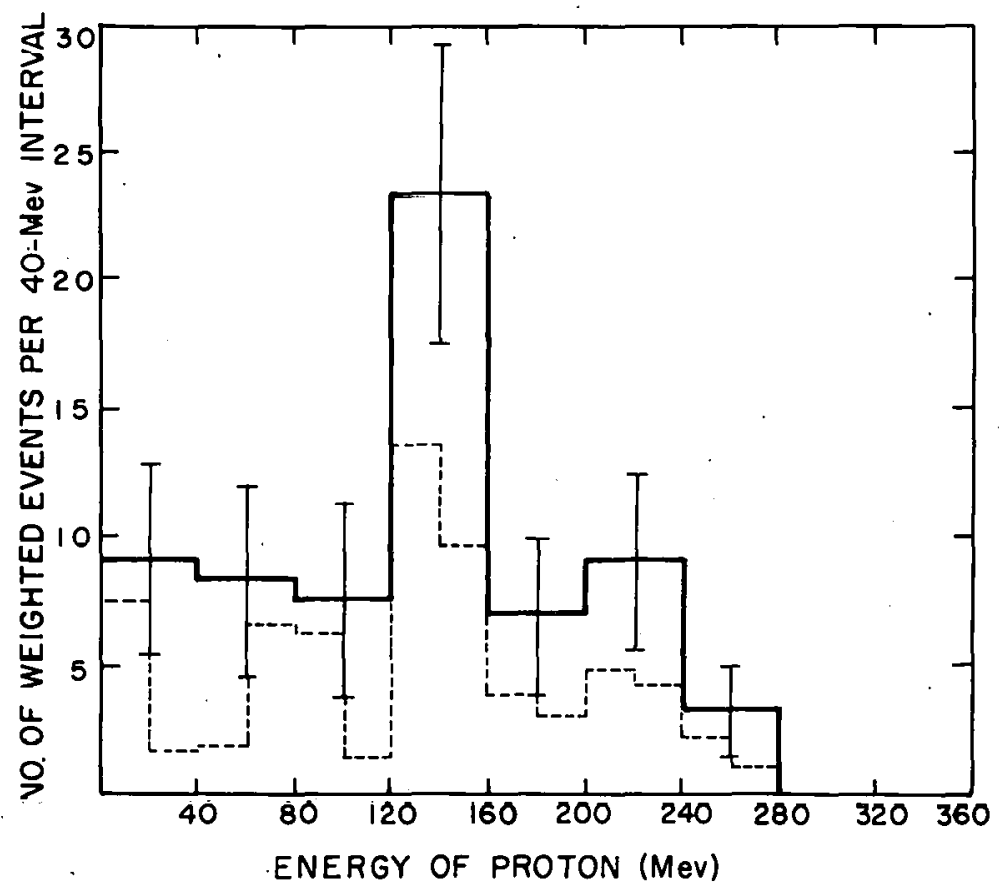

MU-14365

Fig. 8. Energy distribution of protons from $[\mathrm{PD}]$ events. 


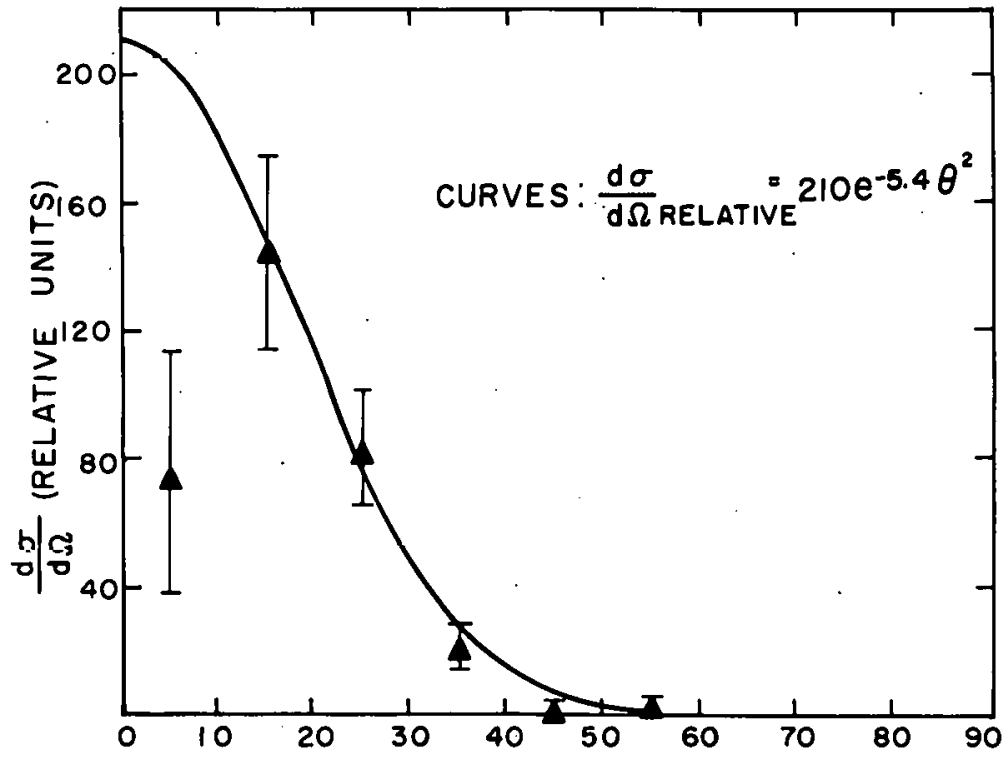

SCATTER ANGLE $\theta$ (deg) (CENTER-OF-MASS SYSTEM)

$M U-14366$

Fig. 9. Angular distribution of elastically scattered neutrons . with energy greater than $160 \mathrm{Mev}$. 


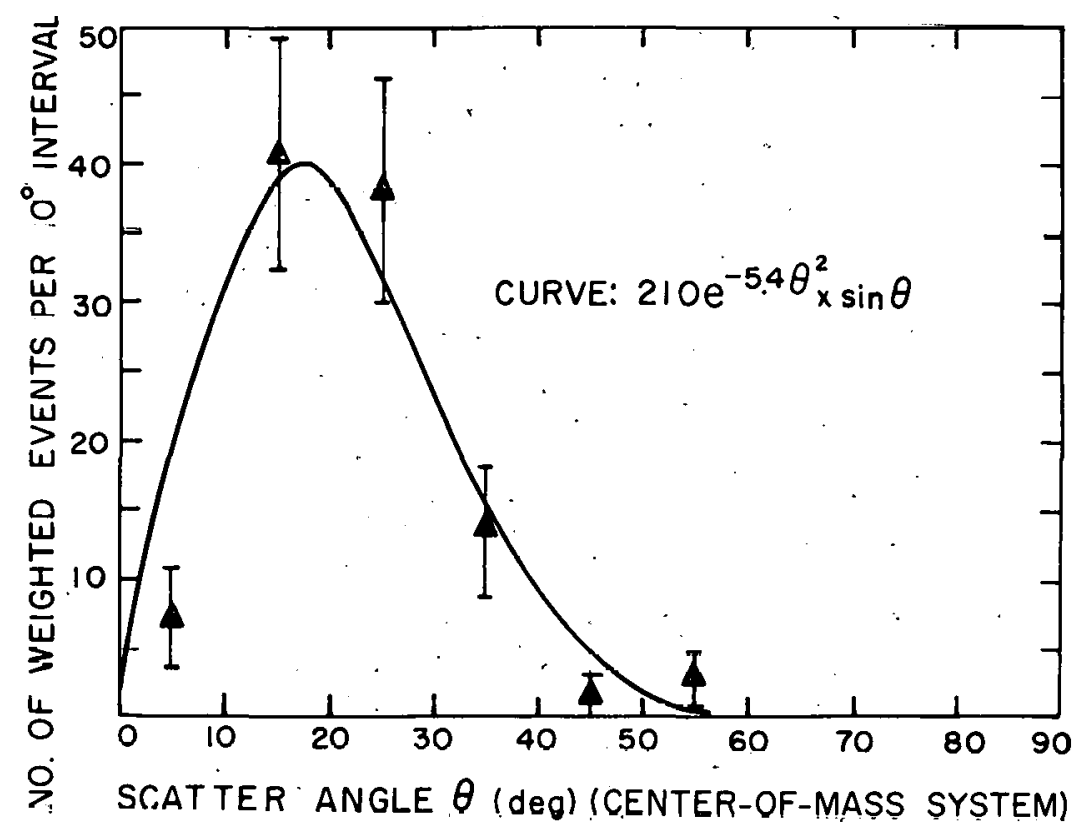

MU-14367

Fig. 10. Angular distribution of ela stically scattered neutrons for incident neutrons with energy greater than $160 \mathrm{Mev}$. 


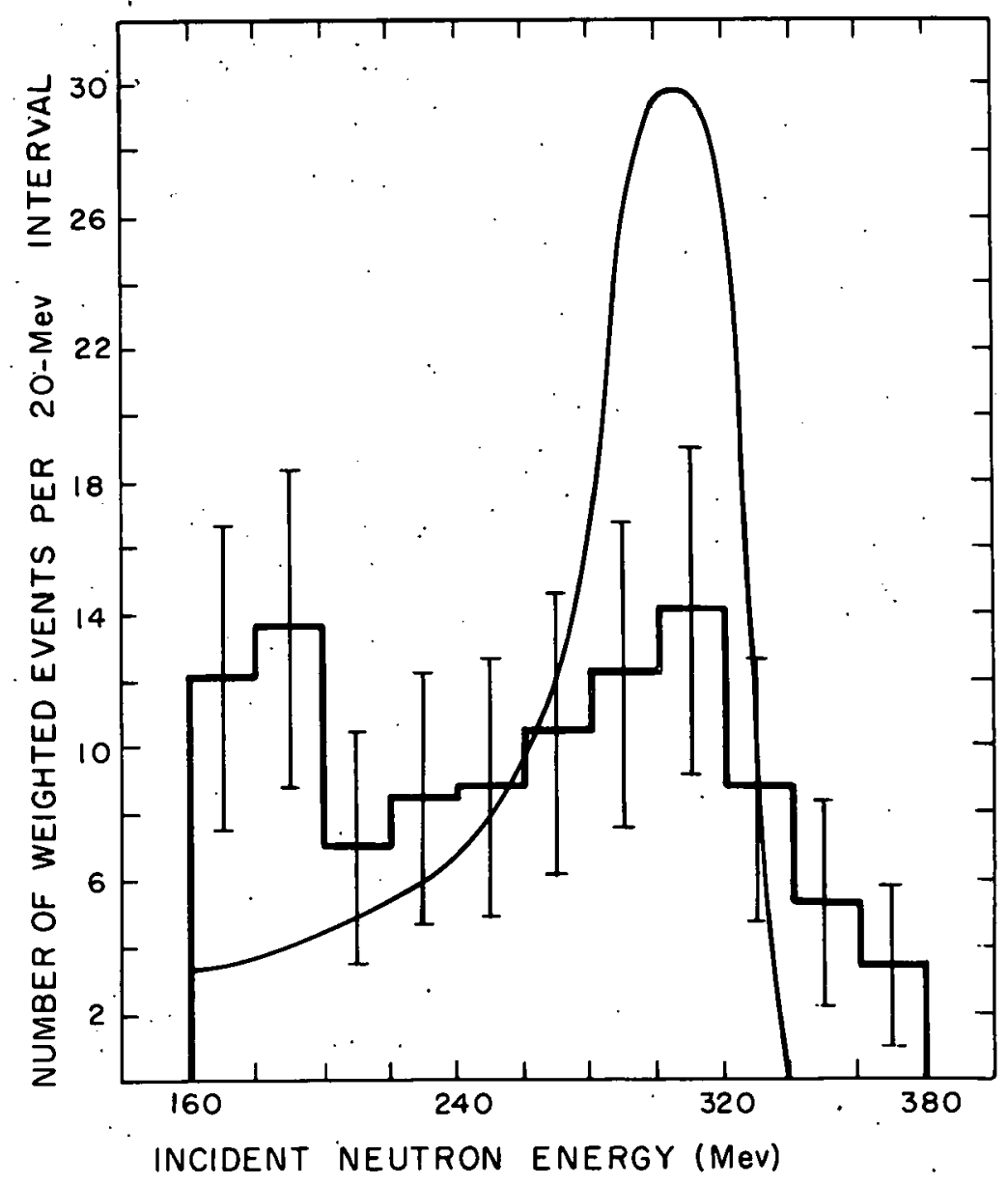

$M U-14368$

Fig. 11. Energy distribution of the incident neutrons, from $\mathrm{He}^{4}$ recoils. The solid curve is De Pangher's neutron energy spectrum. 


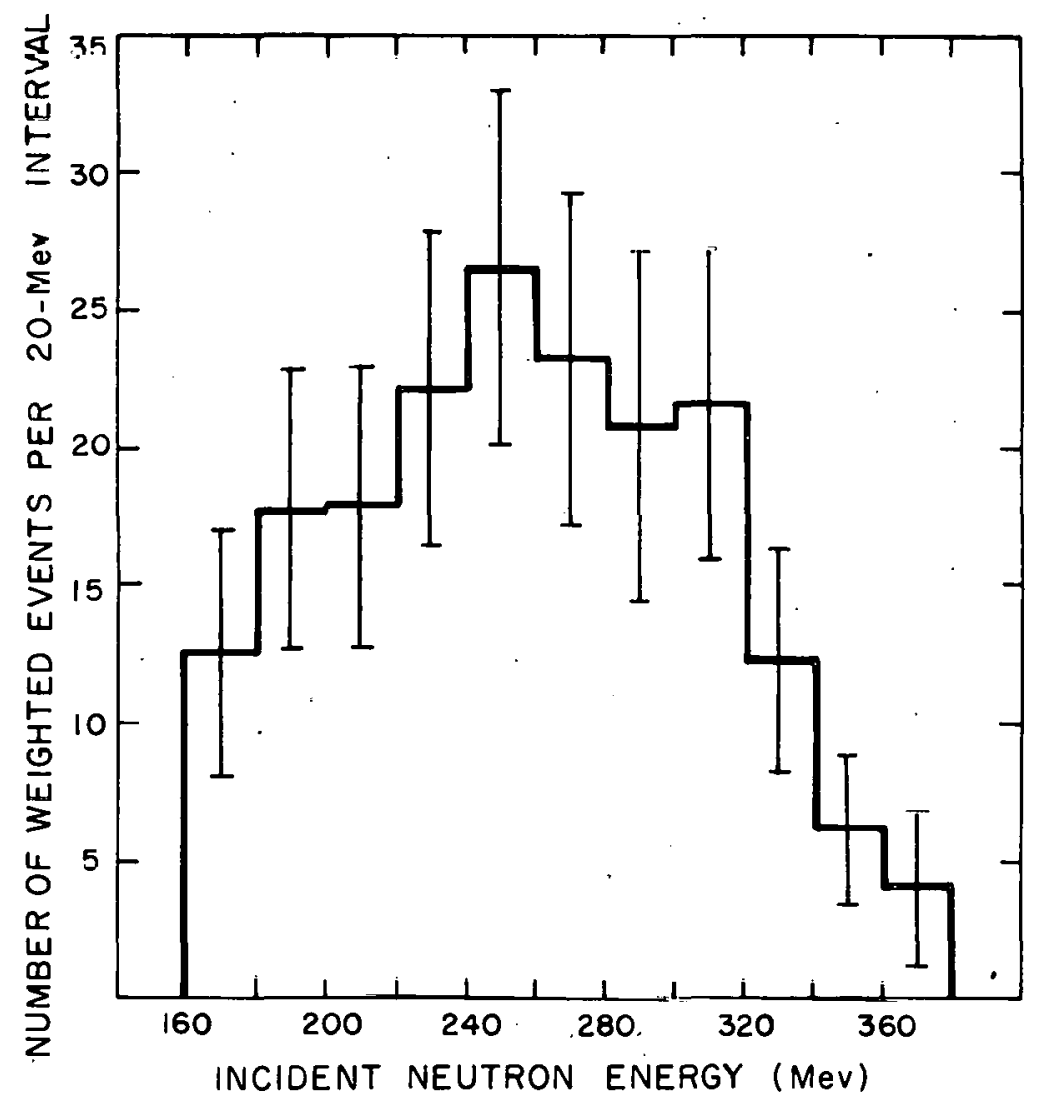

$M U-14369$

Fig. 12. Energy distribution of the incident neutrons from $\angle \mathrm{PT}\rangle, \angle \mathrm{DD}\rangle, \angle \mathrm{DT}\rangle$ events。 


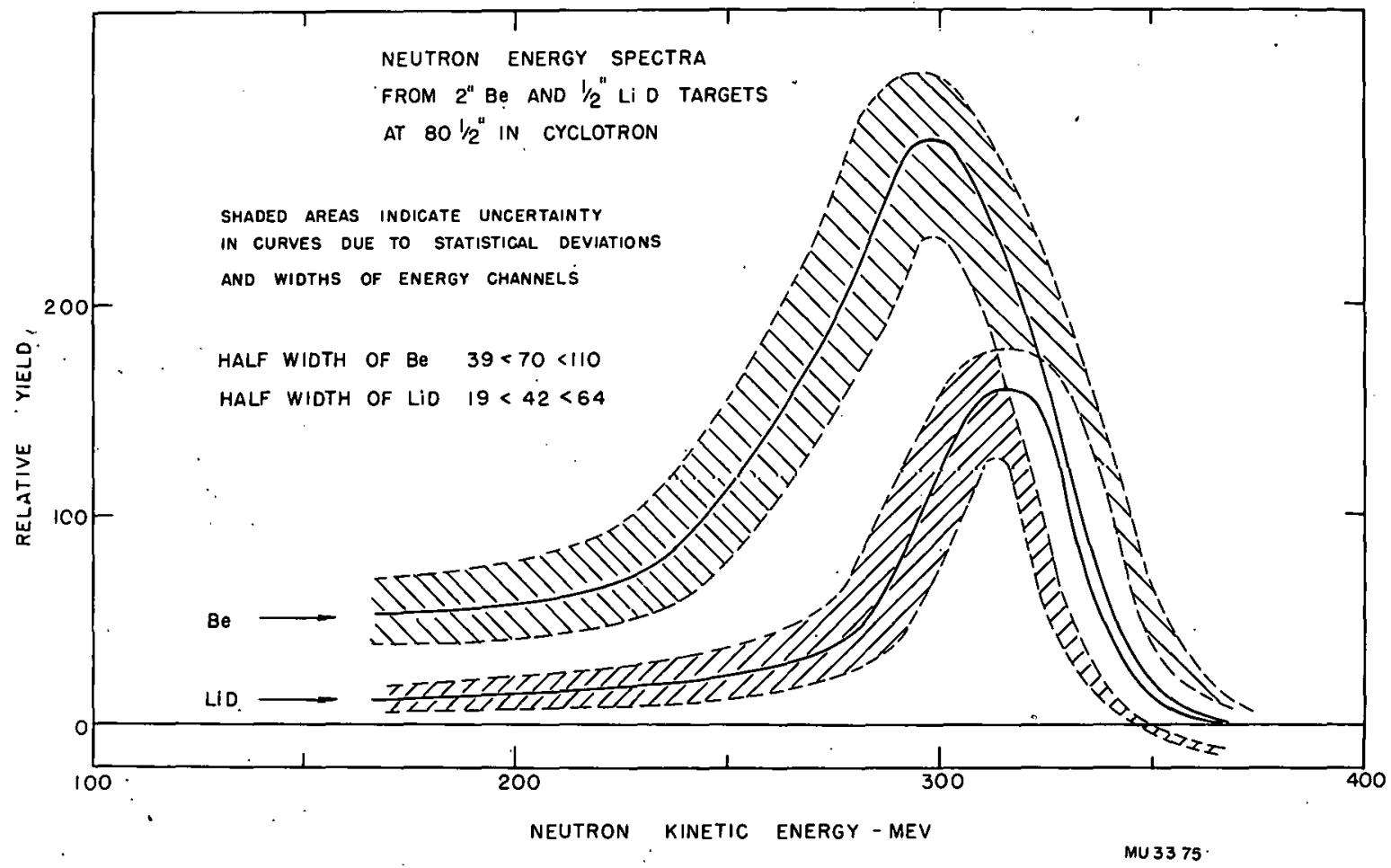

Fig. 13. Energy spectrum of neutrons from a $1 / 2$-inch LiD target. 


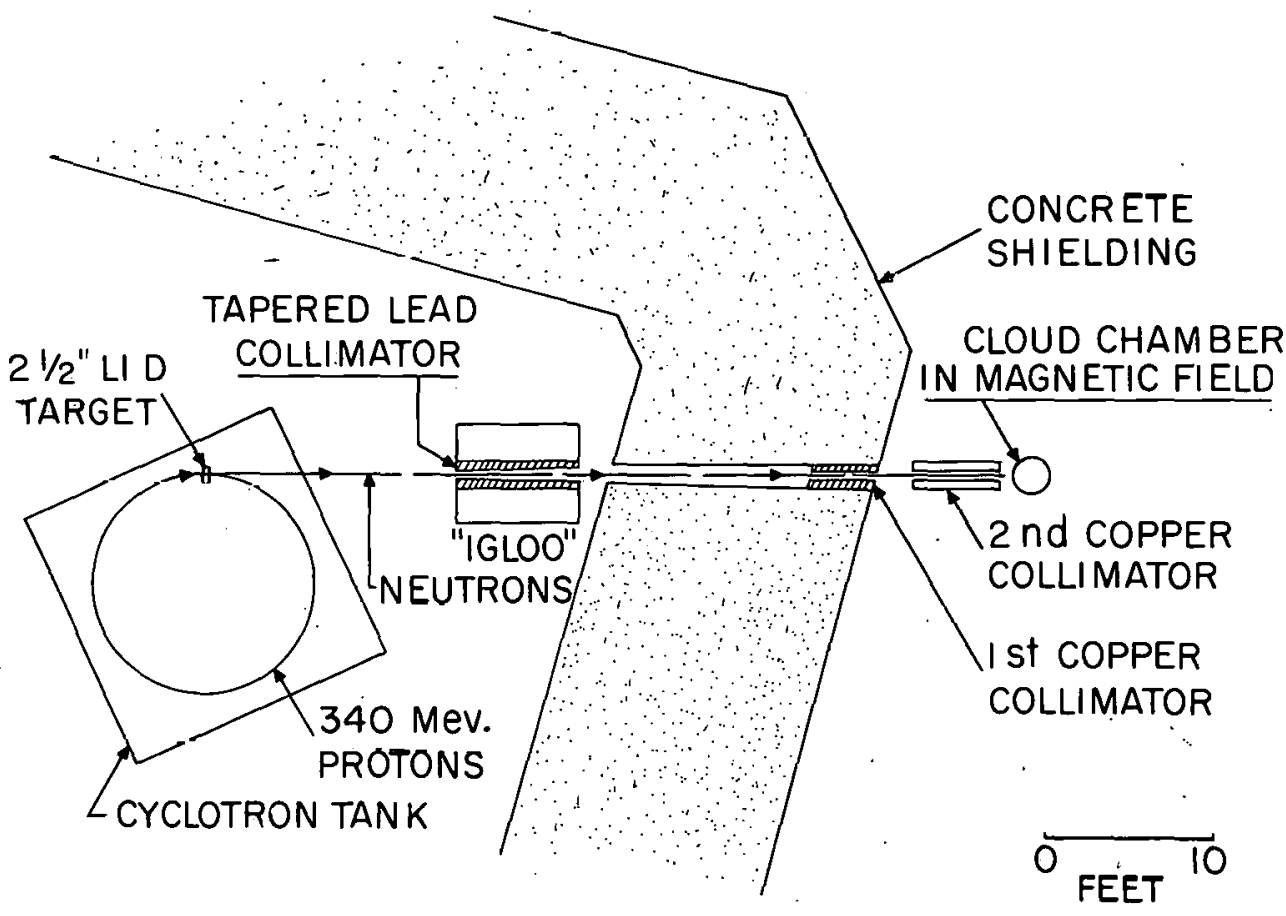

MU-4779-A

Fig. 14. Collimating system. 


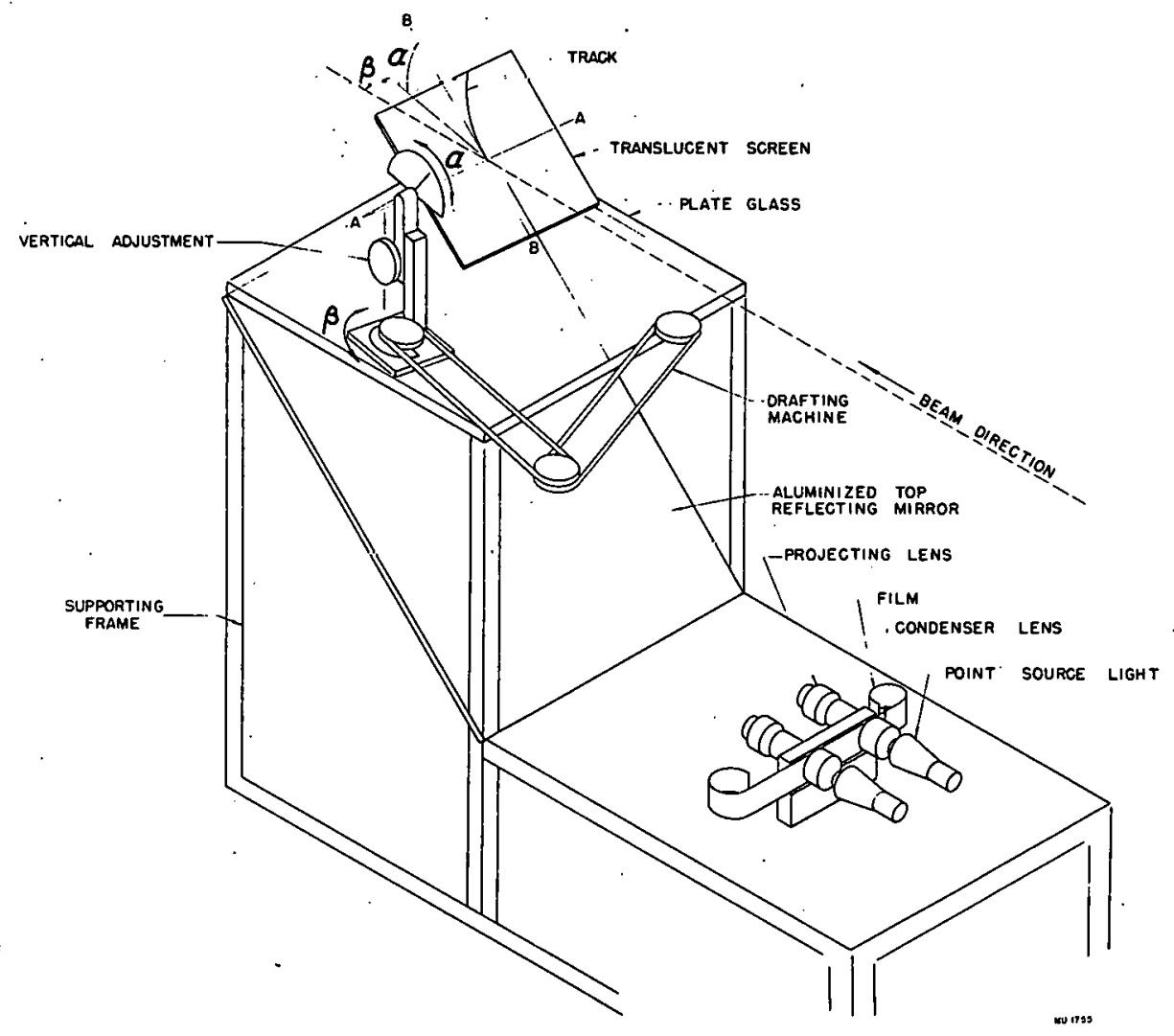

Fig. 15. Reprojection apparatus. 


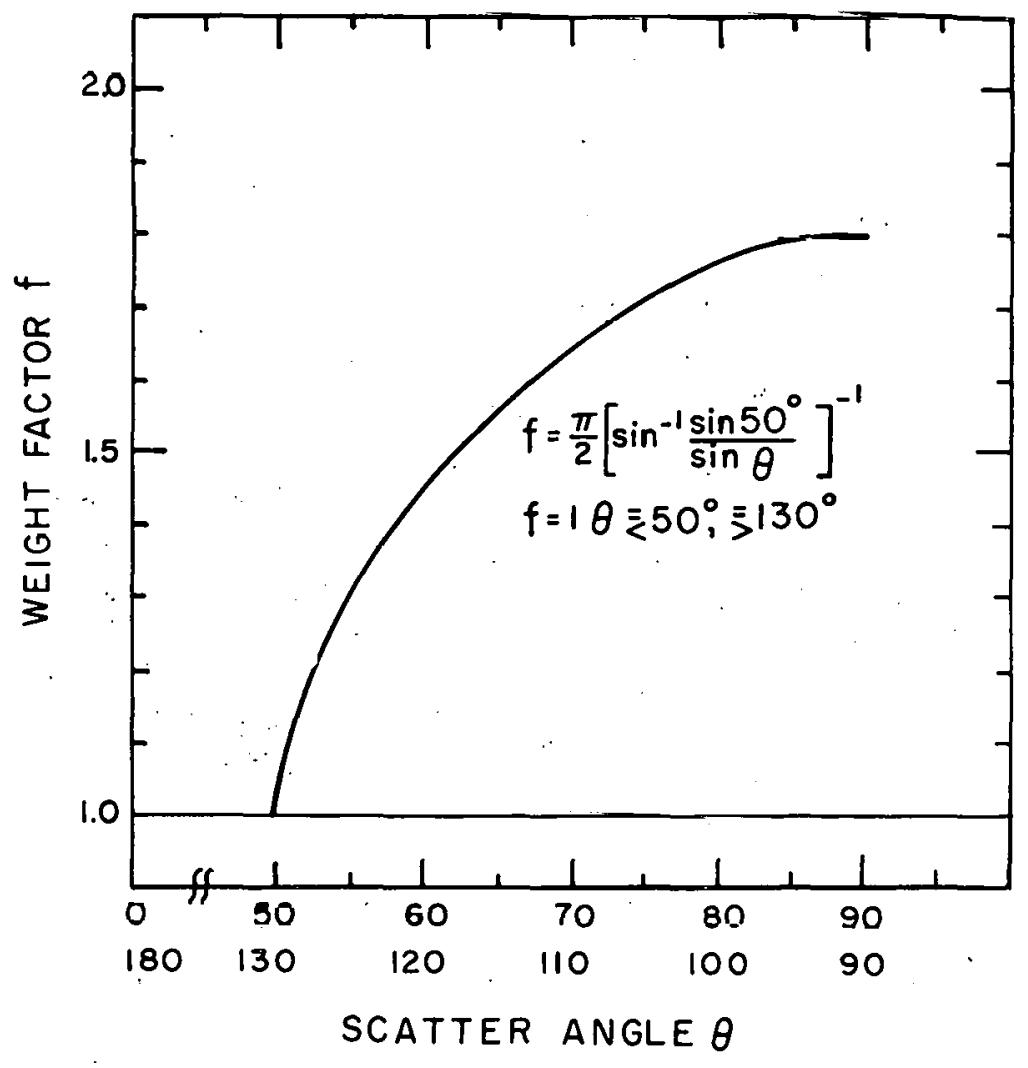

$M U-14370$

Fig. 16. Geometric correction factor for single-track events. 


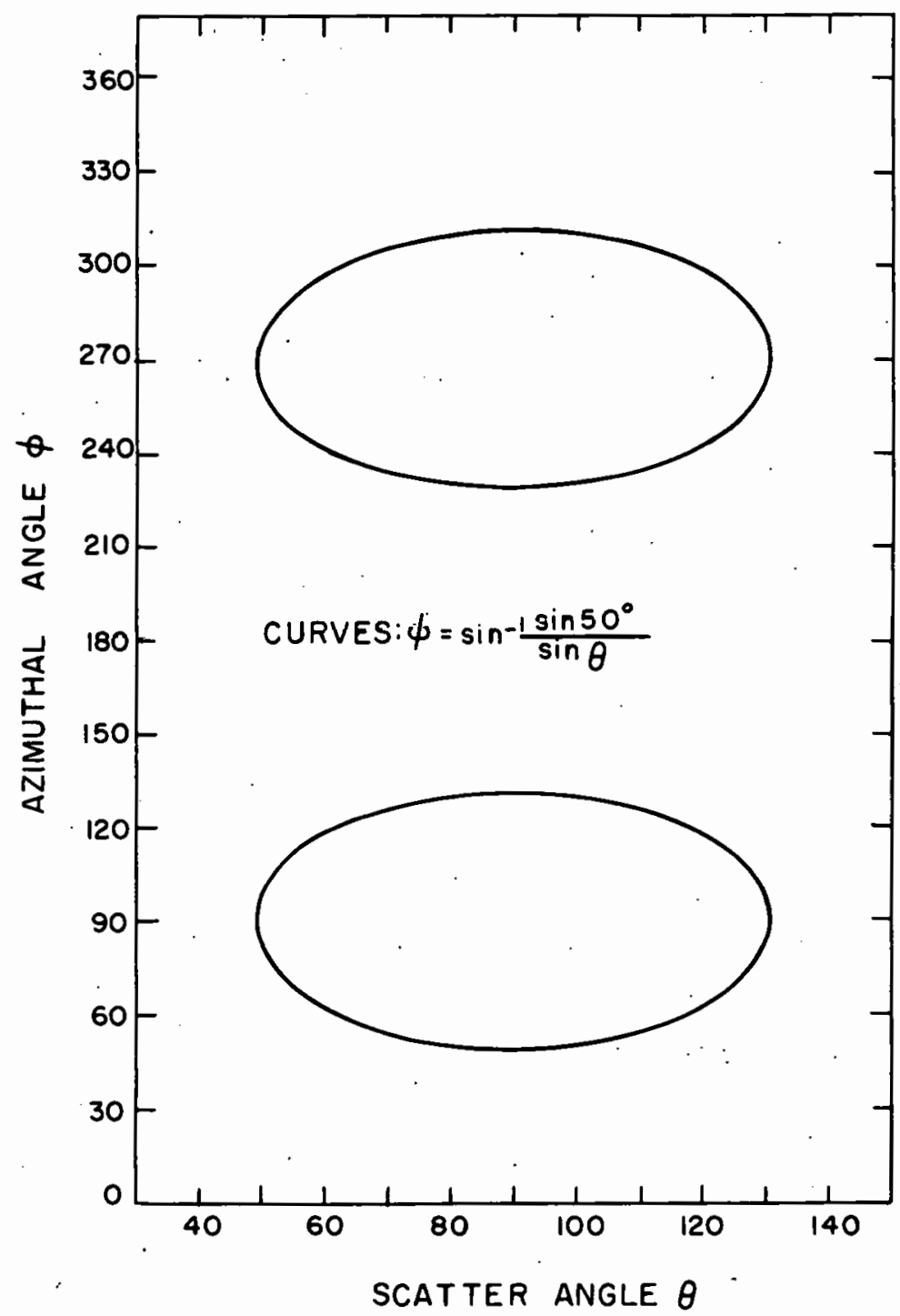

MU-14371

Fig. 17. Geometric correction factor diagram for two-pronged events. 


\section{REFERENCESS}

1. P. T.annenwald, Phys. Rev. 89, 508 (1953).

2. J. Tracy and W.M. Powell, Phys. Rev. 77, 594 (1950).

3. C. Swartz, Phys. Rev. 85, 73 (1952).

4. Hillman, Stahl, and Ramsey, Phys. Rev. 96, 115 (1954).

5. P. H. Moulthrop, Phys. Rev. 99, 1509 (1955).

6. J. Heidmann, Phil. Mag. 41, 444 (1950).”.

7. J. Heidmann, Compt. rend. 234, 1446 (1352).

3. W. M. Powell, Rev. Sci. Instr. 20, 403 (1949).

9. Wils on M. Powell, Scattering of High-Energy Deuterons by $90-\mathrm{Mev}$ Neutron, in UCRL-1191, March 1951 , p. 3.

10. B rueckner, Hartsough, Hayward, and Powell, Phys. Rev. 75, 555 (1949):

11. J.F. Tracy, Cloud Chamber Studies of the Stars in Oxygen (M.S. Thesis), Dept. of Electrical Engineering,: Univ. of California), UCRL-321, April 1949.

12. N. N. Das Gupta and S.K. Ghosh, Revs. Modern Phys. 18, 225 (1946).

13. Donald Johnson, Energy and Ionization of Light Particles as a Function of $\mathrm{H} \rho$, UCRL-1445, Aug: 1951.

14. John De Pangher, Jrs., A High-Pressurc Cloud-Chamber Investigation of Protons Scattered by $300-\mathrm{Mev}$ Neutrons (Thesis) UंCRL-2153, March 1953.

15. M.S. Livingston and H:A. Bethe, Revs. Modern Phys. 9, 261 (1937).

16. W.A. Aron, B.G. Hoffman, and F.C. Williams, Range-Energy Curves, United States Atomic Energy Commission Report No. AECU-663.(U.CRL-121) (2d rev.), 1949.

17. H.A. Bethe, "Range-Energy Curves: Alpha-Particles, Protons and Mesons", United States Atomic Energy Commission Report No. AECU-347, (BNL-T-7), June 1949.

18. A.E. Taylor, Phys. Rev. 92, 1071 (1953). 
19. J. DeJuren, Phys。Rev。 80, 27 (1950).

20. Fox, Leith, Wouters, and MacKenzie, Phys, Rev. 80, 23 (1950).

21. V.A. Nedzel, Phys. Rev. 94, 174 (1954).

22. J. Heidmann, private communication to P. Tannenwald (see Ref. 1).

23. J. Heidmann, private communication to C. Swartz (see Ref. 3).

24. ..J.'. De Juren and B. J. Moyer, Phys. Rev。81, 919 (1951).

25. Ball, Moyer, and Richardson, Phys. Rev. 92, 539 (1953).

26. G.F. Chew and M. L. Goldberger, Phys. Rev. 77, 470 (1950).

27. J. Heidmann, Phys. Rev. 80, 171 (1950)。

28. W. N. Hess and B.J. Moyer, Phys. Rev. 101, 337 (19.56).

29. William P. Ball, Nuclear Scattering of 300 -Mev Neutrons (Thesis), UCRL-1938, Aug. 1952.

30. Millburn, Birnbaum, Crandall, and Schecter, Phys. Rev. 95, 1268 (1954).

31. Fernbach, Serber, and Taylor, Phys. Rev. 75, 1352 (1949).

32. Franklin C. Ford, The Production of Charged Pi Mesons by Neutrons on Oxygen (Thesis), UCRL -2148, March 1953.

33. Myron W. Knapp, Negative Pions from Neutron Bombardment of Deuterons (Thesis), UCRL-2799, Nov. 1954.

34. J. De Pangher, Phys. Rev. 99, 1447 (1955).

35. M. O. Fuller, Phys. Rev. 98, 274 (1955). 